Interfaces and Free Boundaries 13 (2011), 191-222

DOI $10.4171 / \mathrm{IFB} / 254$

\title{
On the Hölder regularity of the landscape function
}

\author{
Alessio Brancolini AND SERGio Solimini \\ Dipartimento di Matematica, Politecnico di Bari, Via Orabona, 4, I-70125 Bari, Italy \\ E-mail: a.brancolini@poliba.it, s.solimini@poliba.it
}

[Received 28 October 2009 and in revised form 11 June 2010]

We study the Hölder regularity of the landscape function introduced by Santambrogio in [S]. We develop a new technique which both extends Santambrogio's result to lower Ahlfors regular measures in general dimension $h$ and simplifies its proof.

Keywords: Optimal transportation problems; irrigation models; landscape function.

\section{Introduction}

In the last decade, much attention has been given to optimal transportation problems. We briefly review here the main definitions and concepts that underlie the subject of the paper.

\subsection{Monge-Kantorovich transportation problem}

This is the original formulation of transportation problems. Given a macroscopic displacement $\left(\mu^{+}, \mu^{-}\right) \in \mathcal{P}\left(\mathbb{R}^{N}\right) \times \mathcal{P}\left(\mathbb{R}^{N}\right)$, the problem consists (in Monge's version) to find the best transport map, i.e. a measurable map $t: \mathbb{R}^{N} \rightarrow \mathbb{R}^{N}$ such that for all Borel sets $B$ we have $\mu^{-}(B)=$ $\mu^{+}\left(t^{-1}(B)\right)$ and which minimizes the cost functional

$$
M(t):=\int_{\mathbb{R}^{N}} c(x, t(x)) \mathrm{d} \mu^{+}(x) .
$$

Usually $c$ is the $p$-th power of the Euclidean distance. The set of transport maps between $\mu^{+}, \mu^{-}$ will be denoted by $\mathcal{M}\left(\mu^{+}, \mu^{-}\right)$.

In Kantorovich's version of the problem, transport maps are replaced by transport plans, i.e. probability measures $\pi \in \mathcal{P}\left(\mathbb{R}^{N} \times \mathbb{R}^{N}\right)$ such that for all Borel sets $A, B \subseteq \mathbb{R}^{N}$ we have $\pi\left(A \times \mathbb{R}^{N}\right)$ $=\mu^{+}(A), \pi\left(\mathbb{R}^{N} \times B\right)=\mu^{-}(B)$, while the functional becomes

$$
K(\pi):=\int_{\mathbb{R}^{N} \times \mathbb{R}^{N}} c(x, y) \mathrm{d} \pi(x, y) .
$$

The set of transport plans between $\mu^{+}, \mu^{-}$will be denoted by $\mathcal{P}\left(\mu^{+}, \mu^{-}\right)$.

As pointed out in [MS1], both the transport maps and transport plans represent a microscopic displacement, that is, a transport map or plan tells us only the initial and final position of a single particle.

We recall that given a transport map $t$ for the macroscopic displacement $\left(\mu^{+}, \mu^{-}\right)$, the measure $\pi_{t}$ given by $\pi_{t}(C):=\mu^{+}\left(\left\{x \in \mathbb{R}^{N}:(x, t(x)) \in C\right\}\right)$ is a transport plan for the same macroscopic displacement and $M(t)=K\left(\pi_{t}\right)$. Kantorovich's problem is thus a generalization 
of Monge's. Actually, it can be seen (see, for example, $[\mathrm{A}-\mathrm{V}]$ or $[\mathrm{V}]$ ) that $K$ is the lower semicontinuous envelope of $M$ with respect to the weak convergence of measures.

Wasserstein spaces. In what follows, we will make use of Wasserstein distances and spaces. Set $c(x, y)=|x-y|^{p}, p \geqslant 1$. The Wasserstein distance of order $p$ between the measures $\mu^{+}, \mu^{-}$is defined by

$$
w_{p}\left(\mu^{+}, \mu^{-}\right):=\left(\min _{\pi \in \mathcal{P}\left(\mu^{+}, \mu^{-}\right)} K(\pi)\right)^{1 / p} .
$$

If $p=+\infty, w_{\infty}\left(\mu^{+}, \mu^{-}\right)$is defined as

$$
w_{\infty}\left(\mu^{+}, \mu^{-}\right):=\min _{\pi \in \mathcal{P}\left(\mu^{+}, \mu^{-}\right)} \pi \text {-ess sup }|x-y| .
$$

Given an open set $X \subseteq \mathbb{R}^{N}$ and $x_{0} \in \bar{X}$, the Wasserstein space of order $p$ over $\bar{X}$ is the set of measures

$$
W_{p}(\bar{X}):=\left\{\mu \in \mathcal{P}\left(\mathbb{R}^{N}\right): \operatorname{spt} \mu \subseteq \bar{X}, w_{p}\left(\mu, \delta_{x_{0}}\right)<+\infty\right\} .
$$

It can be seen that:

- the definition does not depend on $x_{0}$;

- $W_{p}(\bar{X})$ endowed with the Wasserstein distance $w_{p}$ is a complete metric space;

- if $X$ is bounded, convergence with respect to $w_{p}$ is equivalent to weak convergence of measures.

For the details related to the Wasserstein distance see, for example, [V].

\subsection{Irrigation models}

In order to introduce the concept of macroscopic/microscopic motion and of ramified transportation several attempts have been made. Ramified transportation was introduced in the papers by Maddalena, Morel and Solimini ([MMS $]$ ) and by Xia $([\overline{\mathrm{X}}])$. The model proposed by Xia is the relaxation on vector measures of a functional defined on weighted directed graphs. The concept of macroscopic/microscopic motion was introduced by Maddalena and Solimini in [MS1].

In their approach Maddalena, Morel and Solimini consider paths starting from a source and representing the trajectory in $\mathbb{R}^{N}$ of a fluid particle or a fiber of a tree. All the paths start from a common source $S$ and the irrigated measure is defined by counting how many fibers stop in a given volume.

In the original formulation, given a probability space $\left(\Omega, \mathcal{B}(\Omega), \mu_{\Omega}\right)$ (the reference space), a set of fibers with source point $S \in \mathbb{R}^{N}$ is a mapping $\chi: \Omega \times \mathbb{R}_{+} \rightarrow \mathbb{R}^{N}$ satisfying the following assumptions:

- for $\mu_{\Omega}$-a.e. $p \in \Omega$, the map $\chi_{p}(\cdot):=\chi(p, \cdot)$ is 1-Lipschitz;

- for $\mu_{\Omega}$-a.e. $p \in \Omega, \chi_{p}(0)=S$.

Recall that if $\Omega$ is a complete separable metric space of uncountable cardinality and $\mu_{\Omega}$ has no atoms, then $\left(\Omega, \mathcal{B}(\Omega), \mu_{\Omega}\right)$ is isomorphic to the standard space $\left([0,1], \mathcal{B}([0,1]), \mathcal{L}_{\mid[0,1]}^{1}\right)$ (see, for example, Theorem 16 in Chapter 15, Section 5 of $[\mathbb{R}]$ ). In the following, we will always assume that the hypothesis of that result is satisfied.

In this model the irrigating measure is the Dirac mass at the point $S$, i.e. $\mu^{+}=\delta_{S}$, while the irrigated measure is the image of $\mu_{\Omega}$ via the map $p \mapsto i_{\chi}(p):=\chi\left(p, \sigma_{\chi}(p)\right)$, where $\sigma_{\chi}(p)$ is 
the stopping time, i.e. the infimum of $t$ such that $\chi_{p}(\cdot)$ is constant on $[t,+\infty]$. This measure is $\mu^{-}$ (but we will simply write $\mu$ in the following). Then, Maddalena, Morel and Solimini consider the functional

$$
\chi \mapsto J_{\alpha}^{0}(\chi):=\int_{\mathbb{R}_{+}} \int_{M_{t}(\chi)}\left[\mu_{\Omega}\left([p]_{t}^{0}\right)\right]^{\alpha-1} \mathrm{~d} p \mathrm{~d} t,
$$

where $M_{t}(\chi)$ is the set of fibers not stopped at time $t$, and $[p]_{t}^{0}$ is the set of fibers which move together up to time $t$, i.e. if $q \in[p]_{t}^{0}$, then $\chi_{q}(s)=\chi_{p}(s)$ for all $s \in[0, t]$.

Here, and in all models considered in this paper, the irrigated measure $\mu$ will be supposed to be compactly supported. The parameter $\alpha$ belongs to the interval $[0,1[$; in that case the concavity of the model function $|x|^{\alpha}$ gives rise to the branched transportation since moving the mass together as much as possible becomes cheaper.

Let us mention the approach by Bernot, Caselles and Morel in [BCM1]. They consider the functional given by

$$
\chi \mapsto J_{\alpha}^{2}(\chi):=\int_{\Omega} \int_{\mathbb{R}_{+}}\left[\mu_{\Omega}\left([p]_{t}^{2}\right)\right]^{\alpha-1}|\dot{\chi}(p, t)| \mathrm{d} t \mathrm{~d} p,
$$

where $[p]_{t}^{2}:=\{q \in \Omega: \chi(p, t) \in \chi(\{q\} \times I)\}$. In this setting (as in the extended one which will be introduced in the next section), the irrigating and irrigated measure can be chosen arbitrarily among probability measures.

\subsection{Extended setting}

In this paper we will consider the general framework introduced by Maddalena and Solimini in [MS1] and further developed in [MS2], which includes and extends all the formulations from [MMS, BCM1].

DEFINITION 1.1 (Irrigation pattern) Let $I \subseteq \mathbb{R}$ be a generic interval. By irrigation pattern we will mean a measurable function $\chi: \Omega \times I \rightarrow \mathbb{R}^{\bar{N}}$ such that $\chi_{p} \in \mathrm{AC}(I)$ for almost all $p$. The pattern $\tilde{\chi}$ will be equivalent to $\chi$ if the images of $\mu_{\Omega}$ through the maps $p \mapsto \chi_{p}$ and $p \mapsto \tilde{\chi}_{p}$ are the same.

Notation. In the whole paper we will always denote by $a$ (respectively, $b$ ) the infimum (respectively, supremum) of $I$.

DEFINITION 1.2 (Solidarity classes) For every $(p, t) \in \Omega \times I$ we consider the sets

$$
\begin{aligned}
& {[p]_{t}^{0}:=\{q \in \Omega: \chi(q, s)=\chi(p, s), \forall s \in[0, t]\},} \\
& {[p]_{t}^{1}:=\{q \in \Omega: \chi(q, t)=\chi(p, t)\},} \\
& {[p]_{t}^{2}:=\{q \in \Omega: \chi(p, t) \in \chi(\{q\} \times I)\} .}
\end{aligned}
$$

For every $i \in\{0,1\}$ and every $t \in I,\left\{[p]_{t}^{i}: p \in \Omega\right\}$ is a partition of $\Omega$.

The masses $m_{\chi}^{i}$ are given by

$$
m_{\chi}^{i}(p, t):=\mu_{\Omega}\left([p]_{t}^{i}\right)
$$

DEFINITION 1.3 (Cost densities, cost functionals) For $i \in\{0,1,2\}$ we consider the following cost densities:

$$
s_{\alpha}^{i}(p, t):=\left[m_{\chi}^{i}(p, t)\right]^{\alpha-1} .
$$


The cost functionals we are interested in will be

$$
J_{\alpha}^{i}(\chi):=\int_{\Omega \times I} s_{\alpha}^{i}(p, t)|\dot{\chi}(p, t)| \mathrm{d} p \mathrm{~d} t
$$

The variational problem considered in this paper will then be the minimization of $J_{\alpha}^{i}$, given the irrigating and irrigated measure. The irrigating measure will always be the Dirac mass $\delta_{S}$, while the irrigated measure will be denoted by $\mu$ (we will write $\mu_{\chi}$ to stress that it is the measure irrigated by the pattern $\chi$ ). Finally, we will denote by $d_{\alpha}\left(\delta_{S}, \mu\right)$ the least irrigation cost (which is the same for all the functionals as proved in [MS2]).

For $i=0,1$ the functional is synchronous, i.e. if the trajectories of two particles given by an optimal pattern are the same, then the particles will move together. For $i=2$, the functional is asynchronous, since each particle can move independently on its trajectory, i.e. for every $p$ the function $\chi_{p}$ can be reparameterized (independently) without losing the optimality of the reparameterized pattern. $J_{\alpha}^{0}, J_{\alpha}^{1}, J_{\alpha}^{2}$ are respectively the functionals originally introduced in [MMS], [MS1] and [BCM1]. We refer to [MS2] for the proof of the next theorem, which is the fundamental tool to present the unified theory of irrigation functionals.

THEOREM 1.4 (Synchronization Theorem) The following statements hold:

- $J_{\alpha}^{2} \leqslant J_{\alpha}^{1} \leqslant J_{\alpha}^{0}$.

- $J_{\alpha}^{0}, J_{\alpha}^{1}$ share the same minima if the initial mass is a Dirac mass.

- Every optimal pattern for $J_{\alpha}^{2}$ can be reparameterized to be a minimum for $J_{\alpha}^{1}$, i.e. every optimal pattern for $J_{\alpha}^{2}$ is synchronizable (see also $[\overline{\mathrm{BF}}]$ for a proof).

- Every optimal pattern for $J_{\alpha}^{1}$ is optimal for $J_{\alpha}^{2}$.

When we say that $\chi$ is optimal, we always mean that $\chi$ is a minimum for $J_{\alpha}^{1}$ (hence, a minimum of $J_{\alpha}^{0}$, too). Notice that by Theorem 1.4 if a result which involves quantities invariant under scaling (as, for instance, the Santambrogio landscape function introduced in the next section) holds for optimal patterns it must also hold for minima of $J_{\alpha}^{2}$.

\subsection{The landscape function}

The proper subject of this paper is the landscape function introduced by Santambrogio in [S]. The landscape function is, actually, a natural mathematical object to be introduced in the context of branched transportation models. Also, it is connected to the shaping of river basins as many works of geophysicists pointed out (we refer to the introduction of $[\mathrm{S}]$ for a detailed discussion).

Suppose $\chi$ is a optimal pattern for the functional $J_{\alpha}^{2}$ irrigating a measure $\mu$ from a Dirac mass and $\alpha>1-1 / N$. At a point $x=\chi(p, t)$, the landscape function, as introduced by Santambrogio, is defined by

$$
z(x):=\int_{0}^{t}\left[\mu_{\Omega}\left([p]_{t}^{2}\right)\right]^{\alpha-1}\left|\dot{\chi}_{p}(s)\right| \mathrm{d} s .
$$

It is the transportation cost of the mass from the initial source $S$ to the point $x$. The main result of $[\mathbf{S}]$ is the Hölder regularity of the landscape function of exponent $1+N(1-\alpha)$ if the irrigated measure $\mu$ has a density (with respect to the Lebesgue measure) bounded from below by a positive constant and its support is of type $A$, i.e. for every $x_{0} \in$ spt $\mu$ and every $r \in[0$, diam spt $\mu]$ we have $\mathcal{L}^{N}\left(\operatorname{spt} \mu \cap B_{r}\left(x_{0}\right)\right) \geqslant A r^{N}$. As a consequence, the irrigated measure is Ahlfors from below in dimension $N$. 
The object of this paper is to provide a more general definition for the landscape function which will work for a generic pattern and agrees with Santambrogio's definition for optimal ones. In Section 2 we develop a general theory of the landscape function, where it is introduced in a sufficiently general setting to suit the functionals $J_{\alpha}^{0}, J_{\alpha}^{1}, J_{\alpha}^{2}$. For a general pattern (i.e., one that is not optimal) the landscape cannot be introduced directly with an explicit expression like (1.11). We then introduce this function as a sort of relaxation of (1.11).

In Section 3 we develop various type of "gain formulas". These formulas establish how the value of the functional $J_{\alpha}^{0}(\chi)$ varies when we consider a certain variation of the pattern $\chi$. The variation is expressed in terms of a difference of the landscape function values. Combining gain formulas with the results in Section 5 we give estimates on the variation of the landscape between two points of an optimal pattern.

In Section 4 we prove that the Hölder continuity of the landscape function and the decay of the mass on a fiber are related. If the landscape function is Hölder continuous of exponent $\beta$, then the mass $m_{\chi}(x)$ can be estimated from below (up to a constant) by a power $(1-\beta) /(1-\alpha)$ of the residual length of the fiber, and vice versa. Moreover, we prove that Hölder regularity of the landscape function with respect to the distance on fibers is equivalent to Hölder regularity with respect to the Euclidean distance.

In Section 5 we provide an upper estimate for the irrigation cost of a probability measure which satisfies an lower Ahlfors regularity condition (see Definition 5.1).

In Section 6 we extend Santambrogio's result to all Ahlfors measures regular from below in a general dimension $h \geqslant 0$. In Theorem 6.2 we prove that if the irrigated measure $\mu$ satisfies that hypothesis, then the landscape function is Hölder continuous with exponent $1+h(\alpha-1)$. Even though our main result is a generalization of the one in $[\mathbf{S}]$, the proof is completely different and a bit more elementary.

Finally, in Section 8 we provide several examples and counter-examples to show that our results are in a certain sense as sharp as possible. For example, if $\mu$ is Ahlfors regular, then the best Hölder exponent is actually the one provided by Theorem 6.2

For the reader's convenience, the main properties and definitions of the Hausdorff, Minkowski and resolution dimensions are provided in Appendix A. Some technical measurability results are provided in Appendix B A list of the main symbols involved in the paper can be found in Appendix C

\section{Landscape function}

In view of Theorem 1.4, in this section and in the following ones we will consider only the functional $J_{\alpha}^{0}$. We will therefore drop the superscript and write $J_{\alpha}$ for $J_{\alpha}^{0}$.

Consider the $J_{\alpha}$ cost in the extended setting. By the Fubini Theorem, it is the integral on $\Omega$ of

$$
p \mapsto c(p):=\int_{I} s_{\alpha}(p, t)\left|\dot{\chi}_{p}(t)\right| \mathrm{d} t .
$$

$c(p)$ is finite $\mu_{\Omega}$-a.e. $p \in \Omega$ whenever $J_{\alpha}(\chi)<+\infty$. We are thus driven to consider the following definition.

In analogy with the former setting of the problem, we define $i_{\chi}(p):=\chi(p, b)$. Before going on, we introduce the definition of the domain of a pattern, which we will often encounter in the rest of the paper. 
DEFINITION 2.1 (Domain of a pattern) Let $\chi$ be a pattern. The domain of the pattern $\chi$, denoted by $D_{\chi}$, is the set defined by

$$
D_{\chi}=\left\{x: \exists A \subseteq \Omega, \mu_{\Omega}(A)>0, \exists t \in I \forall p \in A, \chi(p, t)=x\right\} .
$$

We now define the landscape function. We remark that in the following we are not supposing that $\chi$ is an optimal pattern, but only a finite cost one, i.e. $J_{\alpha}(\chi)<+\infty$. We will implicitly assume that the pattern $\chi$ has finite cost whenever its landscape function will be considered.

DEFINITION 2.2 (Landscape function) For $\mu_{\Omega}$-a.e. $p$ and all $t \in I$, we define the function $Z_{\chi}$ : $\Omega \times I \rightarrow \mathbb{R}^{N}$ as

$$
Z_{\chi}(p, t):=\int_{a}^{t} s_{\alpha}(p, s)\left|\dot{\chi}_{p}(s)\right| \mathrm{d} s .
$$

A lower semicontinuous function $\varphi$ is admissible for $\chi$ if

$$
\varphi(\chi(p, t)) \leqslant Z_{\chi}(p, t)
$$

for $\mu_{\Omega}$-a.e. $p$ and for all $t \in I$. The landscape function $\bar{Z}_{\chi}$ of the pattern $\chi$ is then defined by

$$
\bar{Z}_{\chi}:=\sup \{\varphi: \varphi \text { admissible for } \chi\} .
$$

REMARK 2.3 Some remarks:

(i) $\bar{Z}_{\chi}$ is lower semicontinuous.

(ii) It is equivalent to require (2.2) for $\mu_{\Omega}$-a.e. $p$ and all $t \in I$ or $\left(\mu_{\Omega} \otimes \mathcal{L}^{1}\right)$-a.e. $(p, t)$ by the continuity of the fibers. Indeed, for any given $p \in \Omega$ consider the set

$$
S_{p}=\left\{t \in I:(\varphi \circ \chi)(p, t)-Z_{\chi}(p, t)>0\right\} .
$$

Let

$$
S=\left\{(p, t) \in \Omega \times I:(\varphi \circ \chi)(p, t)-Z_{\chi}(p, t)>0\right\} .
$$

By the Fubini Theorem,

$$
\left(\mu_{\Omega} \otimes \mathcal{L}^{1}\right)(S)=\int_{\Omega} \mathcal{L}^{1}\left(S_{p}\right) \mathrm{d} p
$$

Thus, $S$ is negligible with respect to $\left(\mu_{\Omega} \otimes \mathcal{L}^{1}\right)$ if and only if $S_{p}$ is negligible for a.e. $p$ with respect to $\mathcal{L}^{1}$.

(iii) $\bar{Z}_{\chi}$ is the maximal 1.s.c. extension on $\bar{D}_{\chi}$ of its restriction to $D_{\chi}$.

(iv) If there is no misunderstanding, we will simply write $\bar{Z}$ instead of $\bar{Z}_{\chi}$.

The next proposition, showing that $\bar{Z}$ is a sort of relaxation of $Z$, follows directly from the definition.

Proposition $2.4 \bar{Z}$ satisfies the inequality

$$
\bar{Z}(\chi(p, t)) \leqslant Z(p, t) \quad \text { for a.e. } p \in \Omega \text { and for all } t \in I \text {. }
$$

Proof. The key point to note is that for a.e. $p$ the quantity $m_{\chi}(p, \cdot)$ is monotone decreasing, so it is sufficient to prove the inequality for a fiber $p$ such that $m_{\chi}(p, t)>0$ for all $t$ in the interior of $I$. Given $\varphi$ admissible, there exists $q \in[p]_{t}$ such that

$$
\varphi(\chi(p, t))=\varphi(\chi(q, t)) \leqslant Z(q, t)=Z(p, t) .
$$

Taking the supremum, we finally get $\bar{Z}(\chi(p, t)) \leqslant Z(p, t)$. 
Proposition 2.5 (Alternative definition of the landscape function) The landscape function $\bar{Z}$ can be characterized as

$$
\bar{Z}(x)=\sup _{r>0} \operatorname{ess} \inf \left\{Z(p, t):(p, t) \in \chi^{-1}\left(B_{r}(x)\right)\right\} .
$$

Proof. The map

$$
x \mapsto \tilde{Z}(x):=\sup _{r>0} \operatorname{ess} \inf \left\{Z(p, t):(p, t) \in \chi^{-1}\left(B_{r}(x)\right)\right\}
$$

is lower semicontinuous, since the set $\{\tilde{Z}>a\}$ is open. Indeed, if for some $x$ we have $\tilde{Z}(x)>a$, then for some $r>0$,

$$
\operatorname{ess} \inf \left\{Z(p, t):(p, t) \in \chi^{-1}\left(B_{r}(x)\right)\right\}>a .
$$

We now prove that $B_{r}(x) \subseteq\{\tilde{Z}>a\}$. For any $x_{1} \in B_{r}(x)$, we have

$$
\operatorname{ess} \inf \left\{Z(p, t):(p, t) \in \chi^{-1}\left(B_{r_{1}}\left(x_{1}\right)\right)\right\}>a
$$

if $B_{r_{1}}\left(x_{1}\right) \subseteq B_{r}(x)$, since

$$
\left\{Z(p, t):(p, t) \in \chi^{-1}\left(B_{r_{1}}\left(x_{1}\right)\right)\right\} \subseteq\left\{Z(p, t):(p, t) \in \chi^{-1}\left(B_{r}(x)\right)\right\},
$$

and ess inf is a decreasing function. By 2.4), $\tilde{Z}\left(x_{1}\right)>a$, so that $B_{r}(x) \subseteq\{\tilde{Z}>a\}$. Since $\tilde{Z}$ is clearly admissible for $\chi$, we must then have $\bar{Z} \leqslant \bar{Z}$.

Let $\varphi$ be any admissible function. Then, by the lower semicontinuity of $\varphi$, for all $x \in \mathbb{R}$,

$$
\begin{aligned}
\varphi(x) & =\sup _{r>0} \inf \left\{\varphi(y): y \in B_{r}(x)\right\} \\
& \leqslant \sup _{r>0} \operatorname{ess} \inf \left\{\varphi(\chi(p, t)):(p, t) \in \chi^{-1}\left(B_{r}(x)\right)\right\} \leqslant \tilde{Z}(x) .
\end{aligned}
$$

Thus, $\varphi \leqslant \tilde{Z}$, which implies $\bar{Z} \leqslant \tilde{Z}$.

REMARK 2.6 If $\bar{Z}(x)<v$, then there exists a sequence $\left(p_{n}, t_{n}\right)$ such that $\chi\left(p_{n}, t_{n}\right) \rightarrow x$ and $Z\left(p_{n}, t_{n}\right)<v$. This is an immediate consequence of Proposition 2.5

We end this section by showing (Theorem 2.8) that, if $\chi$ is optimal, $\bar{Z} \circ \chi=Z$ almost everywhere with respect to the product measure on $\Omega \times I$. To this end, we shall need to employ some estimates which are going to be proved in the next section, obviously without the use of this property.

LEMmA 2.7 Let $\chi: \Omega \times I \rightarrow X$ be an optimal pattern. For a.e. $p \in \Omega$ we have

$$
\bar{Z}(\chi(p, b))=Z(p, b) .
$$

Proof. First of all, note that by Proposition 2.4, $\bar{Z}(\chi(p, b)) \leqslant Z(p, b)$ for a.e. $p \in \Omega$. Suppose moreover that the set

$$
\Omega^{\prime}=\{p \in \Omega: \bar{Z}(\chi(p, b))<Z(p, b)\}
$$

has strictly positive measure. This means that there exist $r, s \in \mathbb{Q}$ such that the set

$$
\Omega^{\prime \prime}=\{p \in \Omega: \bar{Z}(\chi(p, b))<r<s<Z(p, b)\}
$$


has strictly positive measure. Let $X_{j}^{n}$ be a partition of $\mathbb{R}^{N}$ into equal squares with diam $X_{j}^{n}<1 / n$. Since the support of the irrigated measure is compact (and hence bounded), only a finite number of $X_{j}^{n}$ are needed to cover it. Let

$$
\Omega_{j}^{n}:=\Omega^{\prime \prime} \cap i_{\chi}^{-1}\left(X_{j}^{n}\right)
$$

If $\Omega_{j}^{n}$ has positive measure, take $x_{j}^{n}=\chi\left(p_{j}^{n}, t_{j}^{n}\right) \in \mathbb{R}^{N}$ such that (just apply Remark 2.6 to any point $\chi(p, b)$ with $\left.p \in \Omega_{j}^{n}\right)$

$$
d\left(x_{j}^{n}, X_{j}^{n}\right)<1 / n, \quad Z\left(p_{j}^{n}, t_{j}^{n}\right)<r .
$$

The set $\left\{\chi(p, b): p \in \Omega_{j}^{n}\right\}$ (on which $Z>s$ ) is moved to $x_{j}^{n}$. For such a pattern, using the estimates developed in the next section (apply Theorem 3.16 below a finite number of times),

$$
J_{\alpha}\left(\chi_{n}\right)-J_{\alpha}(\chi) \leqslant \alpha \mu_{\Omega}\left(\Omega^{\prime \prime}\right)(r-s) .
$$

Let $\mu, \mu_{n}$ be the measures irrigated by $\chi, \chi_{n}$ respectively. Since $w_{1}\left(\mu_{n}, \mu\right)<1 / n$, by compactness $\chi_{n} \rightarrow \bar{\chi}$ and $\bar{\chi}$ irrigates $\mu$. By lower semicontinuity of the $J_{\alpha}$ cost function (see Lemma 4.6 of [MS1])

$$
J_{\alpha}(\bar{\chi}) \leqslant J_{\alpha}(\chi)+\alpha \mu_{\Omega}\left(\Omega^{\prime \prime}\right)(r-s)<J_{\alpha}(\chi),
$$

which is not possible because of the minimality of $\chi$.

The next theorem shows the equivalence between $\bar{Z}$ and $z$ (defined in the Introduction). The theorem is proved for a minimum of $J^{0}$ and, consequently, for a minimum of $J^{1}$. Since every mimimum of $J^{2}$ can be synchronized with a minimum of $J^{0}$ without changing $z$, the proof of the equivalence is complete.

THEOREM 2.8 Let $\chi: \Omega \times I \rightarrow X$ be an optimal pattern. For a.e. $p \in \Omega$ and all $t \in I$ we have

$$
\bar{Z}(\chi(p, t))=Z(p, t) .
$$

Proof. By Proposition 2.4 we need to prove only that $\bar{Z}(\chi(p, t)) \geqslant Z(p, t)$. Given $T \geqslant 0$, consider the pattern stopped at time $T<b$ given by

$$
\hat{\chi}(p, t):= \begin{cases}\chi(p, t), & 0 \leqslant t \leqslant T, \\ \chi(p, T), & t>T .\end{cases}
$$

We have, for every $p \in \Omega$ and $t \leqslant T$,

$$
Z_{\hat{\chi}}(p, t)=Z_{\chi}(p, t) \text {. }
$$

By Lemma 2.7, for a.e. $p \in \Omega$,

$$
\bar{Z}_{\chi}(\chi(p, T))=\bar{Z}_{\hat{\chi}}(\hat{\chi}(p, T))=Z_{\hat{\chi}}(p, T)=Z_{\chi}(p, T) .
$$

Equation (2.6) is not sufficient to conclude the proof, since the full measure set $\Omega_{T}$ provided by Lemma 2.7 depends on $T$. Suppose that $c(p)<+\infty$. We can fix $\bar{t}>T$ such that $[p]_{\bar{t}}$ has positive measure. If we had

it would follow that

$$
\bar{Z}_{\chi}(\chi(p, T))<Z_{\chi}(p, T)
$$

$$
\bar{Z}_{\chi}(\chi(q, b))<Z_{\chi}(q, b)
$$

for $q \in[p]_{\bar{t}}$. Since $[p]_{\bar{t}}$ is a set of positive measure, (2.7) contradicts Lemma 2.7 
We recall the following definition from [DS1].

DEFINITION 2.9 (Simple patterns) We say that a pattern $\chi$ is simple if all the fibers which share a common point coincide as functions of the time parameter. In other words, if $\chi(p, t)=\chi\left(p^{\prime}, t^{\prime}\right)$, then $t=t^{\prime}$ and $\chi(p, s)=\chi\left(p^{\prime}, s\right)$ for all $s \in[0, t]$. See Definition 6.1 in [DS1].

Recall that any optimal pattern $\chi$ is simple.

If $\chi$ is a simple pattern, the function $Z(p, t)$ does not actually depend on $(p, t)$, meaning that if $x=\chi(p, t)$ then $Z$ depends actually on $x$ (and not on the particular couple $(p, t)$ which realizes $x$ ). This is the content of the following proposition.

Proposition 2.10 Suppose that $\chi$ is a simple pattern (see Definition 2.9. Then, given $x \in D_{\chi}$, the function $Z$ is constant on the set $\chi^{-1}(x)$.

Proof. By the hypothesis of the proposition, for every given $x \in D_{\chi}$ and a.e. $(p, t) \in \chi^{-1}(x)$, the function $s_{\alpha}(p, \cdot)$ does not depend on $p$ on the interval $[0, t]$.

Notation. In view of Proposition 2.10 if $x=\chi(p, t)$, we will write $Z(x)$ instead of $Z(\chi(p, t))$ if $\chi$ is simple.

Under this notation, Theorem 2.8 implies the following corollary.

Corollary 2.11 Suppose that $\chi$ is an optimal pattern. Then $Z=\bar{Z}$ on $D_{\chi}$.

Proof. Let $x \in D_{\chi}$; then $x=\chi(p, t)$ for $p$ in a set of positive measure. For a.e. $p$ we then have

$$
\bar{Z}(x)=Z(\chi(p, t))=Z(x) .
$$

\section{Gain formula}

\subsection{Moving a mass $m$ from a point $x$ to a point $y$}

Recall (see [DS1]) that if $\chi$ is a simple pattern, then the following formula holds:

$$
J_{\alpha}(\chi)=\int_{\mathbb{R}^{N}}\left|P_{\chi}(x)\right|^{\alpha} \mathrm{d} \mathcal{H}^{1}(x),
$$

where $P_{\chi}(x)=[p]_{t}$ whenever $(p, t)$ satisfies $x=\chi(p, t)$. For 3.1) and the proper definition of $P_{\chi}(x)$ we refer to Theorem 9.2 of [DS1].

Consider the following modification of an irrigation pattern $\chi$. In the next part of the section, we will always suppose that $\chi$ is a simple pattern.

DEFINITION 3.1 (Mass function) Suppose $\chi$ is a simple pattern. The mass function is defined as

$$
m_{\chi}(x)=\left|P_{\chi}(x)\right| .
$$

If $x=\chi(p, t)$, we clearly have $m_{\chi}(x)^{\alpha-1}=s_{\alpha}(p, t)$.

We now come to a key definition. We will refer to Figure 11. Suppose that $\chi$ is a simple pattern. Let $x$ and $y$ be points on distinct fibers. These two fibers coincide up to a certain bifurcation point. Let $C_{1}$ (respectively, $C_{2}$ ) be the curve between $x$ (respectively, $y$ ) and the bifurcation of the fibers containing $x$ and $y$. First we remove a mass $m \leqslant m_{\chi}(x)$ from the branch passing through $x$, add it to the branch passing through $y$ up to $y$ (on $C_{2}$ ) through a deviation of the fibers in a set $M \subset \Omega$, such that $\mu_{\Omega}(M)=m$. This pattern will be named "mass deviation" of the pattern $\chi$ and denoted by $\bar{\chi}_{x, y, M}$. 

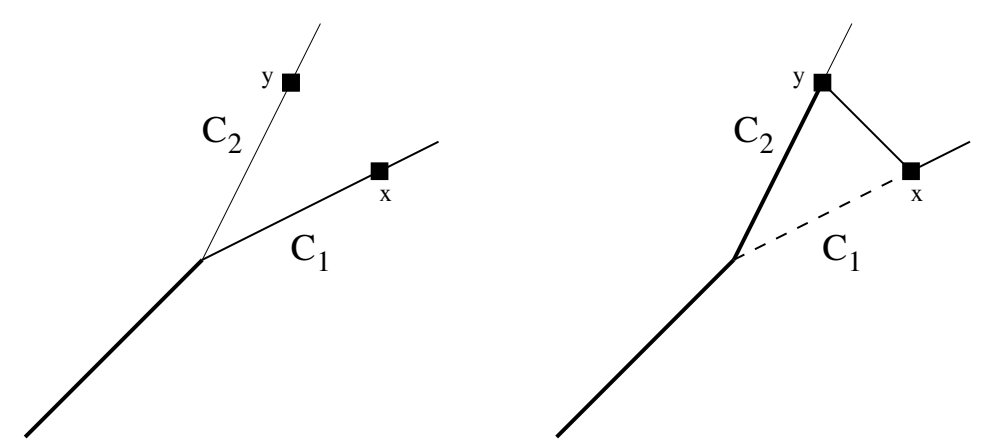

FIG. 1. From the pattern $\chi$ to $\bar{\chi}_{x, y, M}$ and $\tilde{\chi}_{x, y, M}$.

Definition 3.2 (Mass deviation of a pattern $\chi$ ) Suppose that $\chi$ is a simple pattern. Let $x$ and $y$ be points on distinct fibers. Let $x=\chi\left(p_{1}, t_{1}\right)$ and $y=\chi\left(p_{2}, t_{2}\right)$. Let $M \subseteq\left[p_{1}\right]_{t_{1}}$ be such that $\mu_{\Omega}(M)=m$. Define

$$
\bar{\chi}_{x, y, M}(p, t)= \begin{cases}\chi(p, t) & \text { if } p \in \Omega \backslash M, \\ \chi\left(p_{2}, t\right) & \text { if } p \in M, t \leqslant t_{2}, \\ \chi\left(p_{2}, t_{2}\right) & \text { if } p \in M, t \geqslant t_{2} .\end{cases}
$$

We call this new pattern a mass deviation of $\chi$. If there is not ambiguity on $x, y, M$, we will simply write $\bar{\chi}$.

REMARK 3.3 The pattern $\bar{\chi}_{x, y, M}$ does not irrigate the same measure as $\chi$. The irrigated measures are related by

$$
\mu_{\bar{\chi}}=\mu_{\chi \mid(\Omega \backslash M)}+m \delta_{y} .
$$

Suppose that $\chi$ is a simple pattern. Consider the pattern $\bar{\chi}$ of Definition 3.2 If we move the mass deviated to $y$ from $y$ to $x$ along a straight line and recover the irrigated measure we get a new pattern which we call a "mass by-pass" of the pattern $\chi$ and denote it by $\tilde{\chi}_{x, y, M}$. In this way, the original irrigated measure is recovered, i.e. $\mu_{\tilde{\chi}}=\mu_{\chi}$. The way described here, nevertheless, is not the only way of recovering the original irrigated measure.

Definition 3.4 (Mass by-pass of a pattern $\chi$ ) Suppose that $\chi$ is a simple pattern. Let $x$ and $y$ be points on distinct fibers. Let $x=\chi\left(p_{1}, t_{1}\right)$ and $y=\chi\left(p_{2}, t_{2}\right)$. Let $M \subseteq\left[p_{1}\right]_{t}$ be such that $\mu_{\Omega}(M)=m$. Consider the pattern $\bar{\chi}$ of Definition 3.2 and consider the composition of $\bar{\chi}$ with a pattern between $m \delta_{y}$ and $v_{x, y, M}=\mu_{\chi}-\mu_{\bar{\chi}}+m \delta_{y}=\mu_{\chi} \mid M$. We call the new pattern a mass by-pass of $\chi$. If there is not ambiguity on $x, y, M$, we will simply write $\tilde{\chi}$.

REMARK 3.5 The pattern involved in the composition may be the optimal one between $m \delta_{y}$ and $v_{x, y, M}$ or, as is sometimes useful, the pattern built from a straight line between $x$ and $y$ and the optimal one between $m \delta_{x}$ and $v_{x, y, M}$.

REMARK 3.6 The pattern $\tilde{\chi}_{x, y, M}$ irrigates the same measure as $\chi$.

THEOREM 3.7 (First order gain formula, deviation case) Suppose that $\chi$ is a simple pattern. Then the pattern $\bar{\chi}$ satisfies

$$
J_{\alpha}(\bar{\chi})-J_{\alpha}(\chi) \leqslant \alpha m(Z(y)-Z(x)) .
$$


Proof. Recall that by Proposition 2.10 the function $Z$ can be regarded as a function of $x$ instead of $(p, t)$ so that we will write $Z(x)$ for $Z\left(\chi^{-1}(x)\right)$ without confusion.

Let $C_{1}$ and $C_{2}$ be the curves in Definition 3.2 We have

$$
\begin{aligned}
J_{\alpha}(\bar{\chi})-J_{\alpha}(\chi)= & \int_{C_{1}}\left(m_{\chi}(w)-m\right)^{\alpha} \mathrm{d} \mathcal{H}^{1}(w)+\int_{C_{2}}\left(m_{\chi}(w)+m\right)^{\alpha} \mathrm{d} \mathcal{H}^{1}(w) \\
& -\left(\int_{C_{1}} m_{\chi}(w)^{\alpha} \mathrm{d} \mathcal{H}^{1}(w)+\int_{C_{2}} m_{\chi}(w)^{\alpha} \mathrm{d} \mathcal{H}^{1}(w)\right) .
\end{aligned}
$$

The concavity of $u \mapsto u^{\alpha}$ gives

$$
(-m+u)^{\alpha}-u^{\alpha} \leqslant-\alpha m u^{\alpha-1}, \quad(m+u)^{\alpha}-u^{\alpha} \leqslant \alpha m u^{\alpha-1} .
$$

Thus

$$
J_{\alpha}(\bar{\chi})-J_{\alpha}(\chi) \leqslant \alpha m(Z(y)-Z(x)) .
$$

Indeed, we have

$$
Z(y)-Z(x)=\int_{C_{2}} m_{\chi}(w)^{\alpha-1} \mathrm{~d} \mathcal{H}^{1}(w)-\int_{C_{1}} m_{\chi}(w)^{\alpha-1} \mathrm{~d} \mathcal{H}^{1}(w) .
$$

We now consider the mass by-pass case. In the following we will denote by $d_{\alpha}\left(\mu^{+}, \mu^{-}\right)$the least irrigation cost where the initial mass $\mu^{+}$is moved to $\mu^{-}$, i.e.,

$$
d_{\alpha}\left(\mu^{+}, \mu^{-}\right):=\min _{\chi} J_{\alpha}(\chi),
$$

where $\chi$ ranges among the patterns moving $\mu^{+}$on $\mu^{-}$. Recall that convergence with respect to $d_{\alpha}$ is equivalent to weak convergence (see Section 4.2 of [MS1] for the details).

COROLLARY 3.8 (First order gain formula, mass by-pass case) Suppose that $\chi$ is a simple pattern. Then the pattern $\tilde{\chi}$ satisfies

$$
J_{\alpha}(\tilde{\chi})-J_{\alpha}(\chi) \leqslant \alpha m(Z(y)-Z(x))+d_{\alpha}\left(m \delta_{y}, v_{x, y, M}\right) .
$$

COROLlary 3.9 Suppose that $\chi$ is optimal for $J_{\alpha}$. Then

$$
\alpha m(\bar{Z}(x)-\bar{Z}(y)) \leqslant d_{\alpha}\left(m \delta_{y}, v_{x, y, M}\right) .
$$

Proof. Since $\chi$ is optimal and $\tilde{\chi}$ irrigates the same measure, we must obviously have

$$
J_{\alpha}(\tilde{\chi})-J_{\alpha}(\chi) \geqslant 0 .
$$

The conclusion then follows by Corollary 3.8 and Theorem 2.8

COROLlaRY 3.10 Suppose that $\chi$ is optimal for $J_{\alpha}$. Then

$$
\alpha m(\bar{Z}(x)-\bar{Z}(y)) \leqslant m^{\alpha}|x-y| .
$$

Proof. Use as recovery pattern the second one of Remark 3.5 


\subsection{From a set $X$ to a point $y$}

In this part we want to generalize Corollary 3.8 where we replace the point $x$ by a set $X$.

Let us begin with a generalization of Definition 3.2

Definition 3.11 (Mass deviation in the discrete case) Suppose that $\chi$ is a simple pattern. Let $x_{1}, \ldots, x_{n}$ be points with masses $m_{\chi}\left(x_{i}\right) \geqslant m_{i}$ for $i=1, \ldots, n$. A mass deviation of $\chi$ from the set $X=\left\{x_{1}, \ldots, x_{n}\right\}$ to the point $y$ is a pattern given by Definition 3.2 applied iteratively at each point of $X$.

The following lemma is a close generalization of the main theorem of the preceding section.

LEMMA 3.12 (Discrete first order gain formula) Suppose that $\chi$ is a simple pattern. Let $x_{1}, \ldots, x_{n}$ be points with masses $m_{\chi}\left(x_{i}\right) \geqslant m_{i}$ for $i=1, \ldots, n$. Suppose that for $i=1, \ldots, n$ we move the masses $m_{i}$ from $x_{i}$ to a given point $y$. The new pattern $\bar{\chi}$ satisfies

$$
J_{\alpha}(\bar{\chi})-J_{\alpha}(\chi) \leqslant \alpha m\left(Z(y)-\inf _{x \in X} Z(x)\right)
$$

where $m=m_{1}+\cdots+m_{n}$ and $X=\left\{x_{1}, \ldots, x_{n}\right\}$.

Proof. The proof is by induction. For $k \in \mathbb{N}$, set

$$
\Delta_{k}:=Z(y)-\inf \left\{Z\left(x_{1}\right), \ldots, Z\left(x_{k}\right)\right\} .
$$

The statement for $n=1$ is true by Corollary 3.8 . Suppose that the statement is true for the $n-1$ points $x_{1}, \ldots, x_{n-1}$. Let $\bar{\chi}_{n-1}$ be the pattern where the masses at $x_{1}, \ldots, x_{n-1}$ have been moved to $y$. By the inductive hypothesis $\bar{\chi}_{n-1}$ satisfies

$$
J_{\alpha}\left(\bar{\chi}_{n-1}\right)-J_{\alpha}(\chi) \leqslant \alpha\left(m_{1}+\cdots+m_{n-1}\right) \Delta_{n-1} .
$$

Let $Z_{n-1}$ be the landscape function of $\bar{\chi}_{n-1}$. Moving the mass to $x_{n}$, by Corollary 3.8 we get a new pattern $\bar{\chi}$ such that

$$
J_{\alpha}(\bar{\chi})-J_{\alpha}\left(\bar{\chi}_{n-1}\right) \leqslant \alpha m_{n}\left(Z_{n-1}(y)-Z_{n-1}\left(x_{n}\right)\right) .
$$

Clearly, we have $\Delta_{n-1} \leqslant \Delta_{n}$. We just need to prove that

$$
Z_{n-1}(y)-Z_{n-1}\left(x_{n}\right) \leqslant Z(y)-Z\left(x_{n}\right) \leqslant \Delta_{n} .
$$

Inequality (3.6) follows from the fact that, in this and any previous step, moving some mass to the branch containing $y$ does not increase the landscape gap $Z(y)-Z\left(x_{n}\right)$ between $y$ and $x_{n}$.

DEFINITION 3.13 (Fibers passing through a set $X$ ) Given a closed set $X \subseteq \mathbb{R}^{N}$, consider the set of fibers which transit on $X$, i.e. the set

$$
T(X):=\left\{p \in \Omega: \chi_{p}(I) \cap X \neq \emptyset\right\} .
$$

LEMMA 3.14 (Discrete first order gain formula, countable case) Suppose that $\chi$ is a simple pattern. Let $x_{i}, i \in \mathbb{N}$, be points with masses $m_{\chi}\left(x_{i}\right) \geqslant m_{i}$ for $i=1, \ldots, n$. Suppose that we move the masses $m_{i}$ from $x_{i}$ to a given point $y$. The new pattern $\bar{\chi}$ satisfies

$$
J_{\alpha}(\bar{\chi})-J_{\alpha}(\chi) \leqslant \alpha m\left(Z(y)-\inf _{x \in X} Z(x)\right),
$$

where $m=\sum_{i} m_{i}$ and $X=\left\{x_{i}: i \in \mathbb{N}\right\}$. 
Proof. Let $\bar{\chi}_{n}$ be the pattern obtained by moving the $n$ points $x_{1}, \ldots, x_{n}$. Then $\bar{\chi}_{n} \rightarrow \bar{\chi}$ fiberwise and by the lower semicontinuity of the cost functional $J_{\alpha}$,

$$
J_{\alpha}(\bar{\chi}) \leqslant \liminf _{n \rightarrow+\infty} J_{\alpha}\left(\bar{\chi}_{n}\right) \leqslant \liminf _{n \rightarrow+\infty}\left(Z(y)-\inf _{1 \leqslant i \leqslant n} Z\left(x_{i}\right)\right) \leqslant Z(y)-\inf _{X} Z(x),
$$

since, for every $n$,

$$
Z(y)-\inf _{1 \leqslant i \leqslant n} Z\left(x_{i}\right) \leqslant Z(y)-\inf _{X} Z(x) .
$$

REMARK 3.15 In Lemmas 3.12 and 3.14 if we are given a subset $M$ of fibers passing through $X$, i.e. for every $p \in M$ we have $\chi(p, I) \cap X \neq \emptyset$, by Proposition B.3. $M$ can be split into $n$ measurable subsets of fibers passing through $x_{1}, \ldots, x_{n}$ respectively.

We now go on with the last generalization of Theorem 3.7, which we will deduce by a pruning argument from the previous statement.

By Proposition B.4. $T(X)$ is measurable.

Given a subset $M \subseteq T(X)$ such that $\mu_{\Omega}(M)=m$, set

$$
X_{M}:=\left\{x \in X: x \in \chi_{p}(I), p \in S\right\} .
$$

Given a pattern $\chi$, let $\bar{\chi}_{M}$ be the pattern obtained by moving the mass carried by the set of fibers in $M$ to a given $y \in \mathbb{R}^{N}$.

We then have the following theorem.

THEOREM 3.16 (Continuous first order gain formula) Let $\chi$ be a simple pattern. Given a measurable subset $X$ and a subset $M \subseteq X_{M}$ of fibers passing through $X$ such that $\mu_{\Omega}(M)=m$, we have

$$
J_{\alpha}\left(\bar{\chi}_{M}\right)-J_{\alpha}(\chi) \leqslant \alpha m\left(Z(y)-\inf _{X} Z\right) .
$$

Proof. The case $S \in X$ can be easily handled as in the proof of Theorem 3.7. Indeed, using the same notation where $C_{1}$ is no longer the fiber of the point $x$, but the rectifiable set where the mass diminishes, we have

$J_{\alpha}\left(\bar{\chi}_{M}\right)-J_{\alpha}(\chi)=\int_{C_{1}}\left[(m(x)-c(x))^{\alpha}-m(x)^{\alpha}\right] \mathrm{d} \mathcal{H}^{1}(x)+\int_{C_{2}}\left[(m(x)+m)^{\alpha}-m(x)^{\alpha}\right] \mathrm{d} \mathcal{H}^{1}(x)$.

The first term on the r.h.s. is negative, so that

$$
J_{\alpha}\left(\bar{\chi}_{M}\right)-J_{\alpha}(\chi) \leqslant \int_{C_{2}}\left[(m(x)+m)^{\alpha}-m(x)^{\alpha}\right] \mathrm{d} \mathcal{H}^{1}(x) \leqslant \alpha m \int_{C_{2}} m(x)^{\alpha-1} \mathrm{~d} \mathcal{H}^{1}(x) \leqslant \alpha m Z(y) .
$$

This proves the statement of the theorem for $S \in X$ since, in that case, $\inf _{X} Z=0$.

Suppose now that $S \notin X$. For every $\varepsilon>0$, let $X \subseteq V_{\varepsilon}$ be an open subset contained in the $\varepsilon$-neighborhood of $X$ such that

$$
\inf _{V_{\varepsilon}} Z \geqslant \inf _{X} Z-\varepsilon
$$

We can also suppose that $S \notin V_{\varepsilon}$. Except for a null subset of fibers of infinite cost, for every $\bar{p} \in T(X)$ by continuity there exists $\tilde{t} \in \mathbb{Q}$ such that $\chi(\bar{p}, \tilde{t}) \in V_{\varepsilon}$ and $m_{\chi}(\chi(\bar{p}, \tilde{t}))>0$.

Let

$$
F_{t}=\left\{x \in \mathbb{R}^{N}: \exists p \in \Omega, m_{\chi}\left([p]_{t}\right)>0, \chi(p, t)=x\right\} .
$$


Recall that given two fibers $p, q$, we have $[p]_{t}=[q]_{t}$ or $[p]_{t} \cap[q]_{t}=\emptyset$. Given $x_{1}, x_{2} \in F_{t}$, we thus have $x_{1}=\chi\left(p_{1}, t\right)$ and $x_{2}=\chi\left(p_{2}, t\right)$ with $\left[p_{1}\right]_{t} \cap\left[p_{2}\right]_{t}=\emptyset$. Since the total measure of such disjoint solidarity classes cannot exceed one, they must be at most countable. Hence, the set $F_{t}$ is countable.

Consider now the sets

$$
F=\bigcap_{t \in \mathbb{Q}} F_{t}, \quad X_{\varepsilon}=F \cap V_{\varepsilon} .
$$

Then $T(X) \subseteq T\left(X_{\varepsilon}\right)$. Applying Lemma 3.14, we have

$$
J_{\alpha}\left(\bar{\chi}_{M}\right)-J_{\alpha}(\chi) \leqslant \alpha m\left(Z(y)-\inf _{X_{\varepsilon}} Z\right) \leqslant \alpha m\left(Z(y)-\inf _{X} Z\right)+\alpha m \varepsilon .
$$

Since $\varepsilon$ can be chosen arbitrarily, the proof is finished.

Given a pattern $\chi$, let $\tilde{\chi}_{M}$ be the pattern obtained by moving the mass carried by the set of fibers in $M$ to a given $y \in \mathbb{R}^{N}$.

COROllary 3.17 Suppose that $\chi$ is a simple pattern. Let $X \subseteq \mathbb{R}^{N}$ be measurable and $y \in \mathbb{R}^{N}$. Suppose that

$$
Z(y)<\inf _{x \in X} Z(x)
$$

Let $m$ be as in Theorem 3.16 , Then

$$
J_{\alpha}\left(\tilde{\chi}_{M}\right)-J_{\alpha}(\chi) \leqslant \alpha m\left(Z(y)-\inf _{x \in X} Z(x)\right)+d_{\alpha}\left(m \delta_{y}, v\right),
$$

where $v=i_{\chi \#} \mu_{\Omega}-i_{\bar{\chi} \# \mu_{\Omega}}-m \delta_{y}$.

COROLLARY 3.18 Under the same assumptions of Corollary 3.17 if $\chi$ is optimal we have

$$
\alpha m\left(\inf _{x \in X} Z(x)-Z(y)\right) \leqslant d_{\alpha}(y, v)
$$

where $v=i_{\chi} \mu_{\Omega}-i_{\bar{\chi} \# \mu_{\Omega}}-m \delta_{y}$.

Proof. The proof is the same as that of Corollary 3.9

\section{Decay of the multiplicity on a fiber}

DEFINITION 4.1 (Fiber distance) Suppose that $\chi$ is a simple pattern and let $p$ be a fiber. Given two points $x_{1}=\chi_{p}\left(t_{1}\right), x_{2}=\chi_{p}\left(t_{2}\right)$ with $t_{1}<t_{2}$, their fiber distance $d\left(x_{1}, x_{2}\right)$ is given by

$$
d\left(x_{1}, x_{2}\right):=\int_{t_{1}}^{t_{2}}\left|\dot{\chi}_{p}(t)\right| \mathrm{d} t .
$$

Since $\chi$ is a simple pattern, there is a unique fiber between $x$ and $y$ and the integral defining $d\left(x_{1}, x_{2}\right)$ does not depend on $p$. So $d\left(x_{1}, x_{2}\right)$ is well-defined.

Definition 4.2 Let $p$ be a fiber of the pattern $\chi: \Omega \times I \rightarrow \mathbb{R}^{N}$. The function $Z: \mathbb{R}^{N} \rightarrow \mathbb{R}$ is Hölder continuous with exponent $\beta$ with respect to the fiber distance if, for some constant $C$,

$$
\left|Z\left(x_{2}\right)-Z\left(x_{1}\right)\right| \leqslant C d\left(x_{1}, x_{2}\right)^{\beta}
$$

for all $x_{1}=\chi_{p}\left(t_{1}\right), x_{2}=\chi_{p}\left(t_{2}\right)$ 
THEOREM 4.3 Let $\chi$ be a simple pattern, let $Z$ be as in Definition 2.2 and let $p \in \Omega$ be given. Then the following conditions are equivalent:

(i) $Z$ is Hölder continuous with exponent $\beta$ on the fiber $p$ (formula (4.2p);

(ii) the Hölder continuity condition holds when one of the points is a terminal point, i.e. if $x=$ $\chi(p, t)$, we have

$$
Z(\chi(p, b))-Z(x) \leqslant C d\left(x, i_{\chi}(p)\right)^{\beta}
$$

for some constant $C>0$;

(iii) if $x=\chi(p, t)$, then

$$
m(x) \geqslant C d\left(x, i_{\chi}(p)\right)^{\frac{1-\beta}{1-\alpha}}
$$

for some constant $C>0$.

Proof. The proof is divided into three steps.

(i) $\Rightarrow$ (ii). Obvious.

(ii) $\Rightarrow$ (iii). Recall that the function $s \mapsto m(\chi(p, s))$ is non-increasing. Thus,

$$
\begin{aligned}
C d\left(x, i_{\chi}(p)\right)^{\beta} & \geqslant Z\left(i_{\chi}(p)\right)-Z(x)=\int_{t}^{b} m(\chi(p, s))^{\alpha-1}\left|\dot{\chi}_{p}(s)\right| \mathrm{d} s \\
& \geqslant m(\chi(p, t))^{\alpha-1} \int_{t}^{b}\left|\dot{\chi}_{p}(s)\right| \mathrm{d} s=m(x)^{\alpha-1} d\left(x, i_{\chi}(p)\right) .
\end{aligned}
$$

(iii) $\Rightarrow$ (i). Consider the reparameterized fiber, so that $\left|\dot{\chi}_{p}(t)\right|=1$. Let $l$ be its length. From 4.4,

$$
m\left(\chi_{p}(s)\right)^{\alpha-1} \leqslant C|l-s|^{\beta-1} .
$$

Integrating between $t_{1}<t_{2}$, we get

$$
\begin{aligned}
Z\left(\chi_{p}\left(t_{2}\right)\right)-Z\left(\chi_{p}\left(t_{1}\right)\right) & =\int_{t_{1}}^{t_{2}} m(\chi(p, s))^{\alpha-1} \mathrm{~d} s \\
& \leqslant \int_{t_{1}}^{t_{2}} C(l-s)^{\beta-1} \mathrm{~d} s=C\left[\frac{\left(l-t_{1}\right)^{\beta}}{\beta}-\frac{\left(l-t_{2}\right)^{\beta}}{\beta}\right] .
\end{aligned}
$$

By subadditivity $\left(l-t_{1}\right)^{\beta}-\left(l-t_{2}\right)^{\beta} \leqslant\left(t_{2}-t_{1}\right)^{\beta}$, so

$$
Z\left(x_{2}\right)-Z\left(x_{1}\right) \leqslant \frac{C}{\beta}\left(t_{2}-t_{1}\right)^{\beta}=\frac{C}{\beta} d\left(x_{1}, x_{2}\right)^{\beta} .
$$

REMARK 4.4 If $C$ is the constant appearing in inequality (4.2), then our computation provides $C^{1 /(\alpha-1)}$ as the constant in inequality (4.4).

THEOREM 4.5 Let $\chi$ be an optimal pattern. Then the uniform Hölder continuity on the fibers of the landscape function (Definition 4.2 implies its Hölder continuity with respect to the Euclidean distance on $\bar{D}_{\chi}$. 


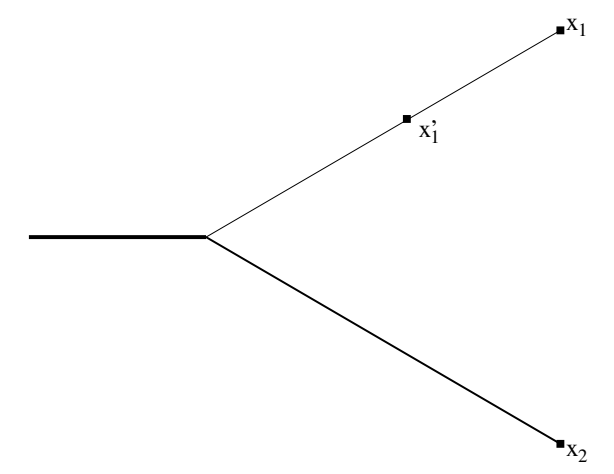

FIG. 2. Proof of Theorem 4.5

Proof. Fix $x_{1}, x_{2}$, let $d=\left|x_{1}-x_{2}\right|$. Suppose first you can take $x_{1}^{\prime}$ backward on the fiber of $x_{1}$ at a fiber distance $d$ (see Figure 2). Thanks to the decay inequality (4.4) of Theorem 4.3, we have

$$
m\left(x_{1}^{\prime}\right) \geqslant C d^{\frac{1-\beta}{1-\alpha}}, \quad \text { that is, } \quad m\left(x_{1}^{\prime}\right)^{\alpha-1} \leqslant C d^{\beta-1} .
$$

By Corollary 3.10 ,

$$
\alpha\left(\bar{Z}\left(x_{1}^{\prime}\right)-\bar{Z}\left(x_{2}\right)\right) \leqslant m\left(x_{1}^{\prime}\right)^{\alpha-1}\left|x_{1}^{\prime}-x_{2}\right| \leqslant\left(C d^{\beta-1}\right)(2 d)=2 C d^{\beta} .
$$

Finally, since $\bar{Z}\left(x_{1}\right)-\bar{Z}\left(x_{2}\right)=\left(\bar{Z}\left(x_{1}\right)-\bar{Z}\left(x_{1}^{\prime}\right)\right)+\left(\bar{Z}\left(x_{1}^{\prime}\right)-\bar{Z}\left(x_{2}\right)\right)$ and each of the two terms is bounded by some constant times $d^{\beta}$ (the first thanks to the Hölder continuity on the fiber and to the equality $Z=\bar{Z} \circ \chi$ implied by the minimality of $\chi$ ), the first part of the proof is complete on $D_{\chi}$ and, by a continuity argument, on $\overline{D_{\chi}}$ (see Remark 2.3 (iii)).

Suppose finally that $x_{1}^{\prime}$ cannot be taken at a fiber distance $d$. Then we can take $x_{1}^{\prime}=S$ and the term $\bar{Z}\left(x_{1}^{\prime}\right)-\bar{Z}\left(x_{2}\right)$ can be estimated by zero.

REMARK 4.6 Note that if the Hölder continuity constant with respect to fibers is $C$, then the constant with respect to the Euclidean distance is at most $C(1+2 / \alpha)$.

\section{Irrigation cost of a measure $\mu$ with a given Ahlfors dimension}

Before entering the last part of the proof of the main result, we prove an estimate from above of the irrigation cost between a Dirac mass and a measure Ahlfors regular from below. The irrigation cost is bounded by the $\alpha$-power of the mass of the irrigated measure times the diameter of its support times a universal constant. We recall that the definition and a complete treatment of the main tool used here, the hierarchy of collectors, can be found in [DS2] (see, in particular, Definition 3.1 and Corollary 3.1 there).

DEFINITION 5.1 (Lower Ahlfors regular measure) A measure $\mu$ is Ahlfors regular from below in dimension $h$ if there exists $C_{A}>0$ such that

$$
\mu(B(x, r)) \geqslant C_{A} r^{h}
$$

for all $r \in[0,1]$ and for all $x \in \operatorname{spt} \mu$. 
A dyadic cube of order $n$ in $\mathbb{R}^{N}$ is a Cartesian product of $N$ intervals of the kind $\left[k 2^{-n},(k+1) 2^{-n}[\right.$ for $k \in \mathbb{Z}, n \in \mathbb{N}$ :

$$
Q_{k_{1}, \ldots, k_{N}}^{n}:=\prod_{i=1}^{N}\left[k_{i} 2^{-n},\left(k_{i}+1\right) 2^{-n}[;\right.
$$

the center of $Q_{k_{1}, \ldots, k_{N}}^{n}$ is the point

$$
c\left(Q_{k_{1}, \ldots, k_{N}}^{n}\right):=\left(2^{-n}\left(k_{1}+1 / 2\right), \ldots, 2^{-n}\left(k_{N}+1 / 2\right)\right) .
$$

Lemma 5.2 Let $B$ be a ball of radius 1 and let $\mu \in \mathcal{P}\left(\mathbb{R}^{N}\right)$ be such that spt $\mu \subseteq \bar{B}$. Suppose also that $\mu$ is Ahlfors regular from below in dimension $h$. Let $P_{n}$ be the set of centers of the dyadic squares of order $n$ which meet spt $\mu$. Then

$$
\# P_{n} \leqslant C_{A}^{-1} 2^{h(n+1)} .
$$

Proof. Consider the balls whose center is the center of some dyadic square of order $n$ and whose radius is $r=2^{-(n+1)}$. Their mass (with respect to the measure $\mu$ ) is at least (by the Ahlfors regularity from below of $\mu) C_{A} 2^{-h(n+1)}$. Moreover, such balls are disjoint. We then have

$$
\left(\# P_{n}\right) C_{A} 2^{-h(n+1)} \leqslant \sum_{x \in P_{n}} \mu\left(B_{2^{-(n+1)}}(x)\right) \leqslant \mu\left(\bigcup_{x \in P_{n}} B_{2^{-(n+1)}}(x)\right) \leqslant 1,
$$

since the total mass is 1 . This inequality gives the conclusion.

THEOREM 5.3 Let $B$ be a ball of radius 1 and let $\mu \in \mathcal{P}\left(\mathbb{R}^{N}\right)$ be such that spt $\mu \subseteq \bar{B}$. Suppose also that $\mu$ is Ahlfors regular from below in dimension $h$. Then, given $\alpha>1-1 / h$ and $S \in B$, we have

$$
d_{\alpha}\left(\mu, \delta_{S}\right) \leqslant C\left(C_{A}, h, N, \alpha\right)<+\infty .
$$

Proof. Without loss of generality, we can suppose that $S$ is the center of $B$. A different source can be managed using a pattern which initially moves the mass from the source to the center of $B$. $C\left(C_{A}, h, N, \alpha\right)$ will be increased at most by 1 , the cost of transportation of a Dirac mass from the boundary of $B$ to its center.

Since spt $\mu$ is compact, for every $n$ we can define $P_{n}$ as in Lemma 5.2. Set $P_{0}=\{S\}$. Consider any dependence map $\gamma_{n}: P_{n} \rightarrow P_{n-1}$ which maps a point in $P_{n}$ to a point in $P_{n-1}$ whose distance does not exceed $2^{-n+1}$, and the corresponding hierarchy of collectors $\left(P_{n}, \gamma_{n}\right)_{0 \leqslant n \leqslant N_{\max }}$.

The cost of the pattern connecting $\delta_{S}$ to $\mu$ is then estimated by (we refer to Corollary 3.3 of [DS2] and to Lemma 5.2]

$$
\sum_{n=1}^{+\infty} 2^{-n+1} \sqrt{N}\left(\# P_{n}\right)^{1-\alpha} .
$$

So $C\left(C_{A}, h, N, \alpha\right)$ can be chosen as

$$
C\left(C_{A}, h, N, \alpha\right)=\frac{4 \sqrt{N} C_{A}^{\alpha-1}}{1-2^{-1+h(1-\alpha)}} .
$$


COROLlary 5.4 Let $\mu \in \mathcal{P}\left(\mathbb{R}^{N}\right)$ be such that $\mu\left(\mathbb{R}^{N}\right)=m$. Suppose that spt $\mu$ is contained in a ball of radius $d$. Suppose also that $\mu$ is Ahlfors regular from below in dimension $h$. Then, given $\alpha>1-1 / h$ and $S \in \mathbb{R}^{N}$,

$$
d_{\alpha}\left(\mu, \delta_{S}\right) \leqslant C\left(C_{A}, h, N, \alpha\right) m^{\alpha} d .
$$

Proof. The proof is the same as that of Theorem 5.3. Note that scaling the length by a factor $d$ multiplies the cost by $d$. A mass scaling by a factor $m$ multiplies the cost by $m^{\alpha}$.

\section{Hölder continuity of the landscape function}

In this section we are going to prove that inequality 4.3 holds for a.e. $p \in \Omega$ with

$$
\frac{1-\beta}{1-\alpha}=h, \quad \text { that is, } \quad \beta=1+h(\alpha-1),
$$

under the hypothesis that the irrigated measure is Ahlfors regular from below in dimension $h$.

LEMMA 6.1 Let $\mu$ be the irrigated measure, which is supposed to be Ahlfors regular from below in dimension $h$. Let $\bar{Z}$ be the landscape function associated to the optimal pattern $\chi$. Let $\beta:=$ $1+h(\alpha-1)$. Then, for some $c>0$,

$$
\bar{Z}\left(x_{0}\right)-\bar{Z}(x) \leqslant c\left|x_{0}-x\right|^{\beta}
$$

for $x_{0}$ in the support of the irrigated measure $\mu$ and $x \in \mathbb{R}^{N}$.

Proof. For contradiction, fix $x, x_{0}$ with $x_{0} \in$ spt $\mu$ such that, for a suitably large constant $c$ (see Figure 3,

$$
Z\left(x_{0}\right)-Z(x)>c\left|x_{0}-x\right|^{\beta} \text {. }
$$

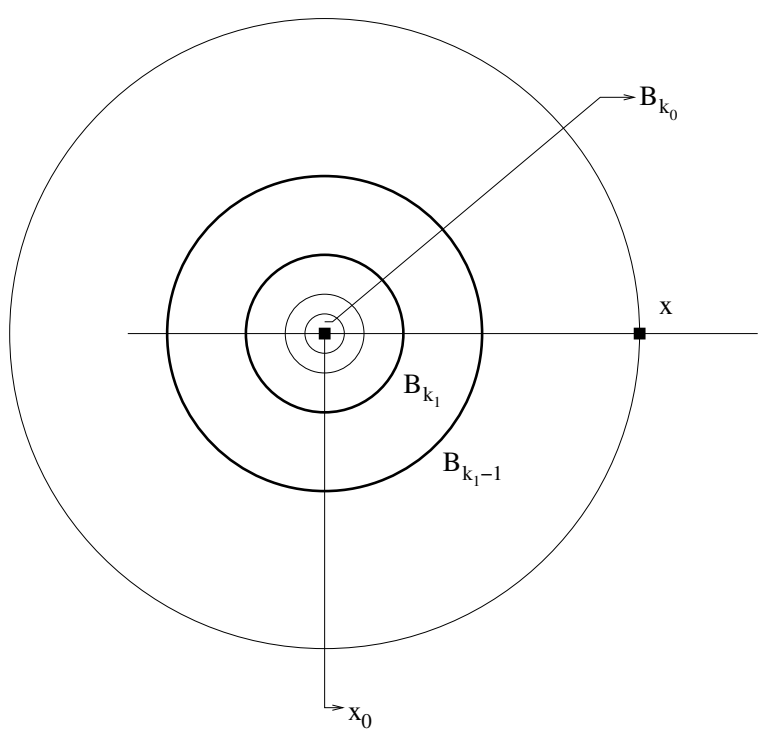

FIG. 3. Proof of Lemma6.1 
Let $r=\left|x_{0}-x\right|$, so $Z\left(x_{0}\right)-Z(x)>c r^{\beta}$. Since we are assuming spt $\mu$ is bounded, we can suppose that $r \leqslant 1$. Indeed, if 6.1 held for all couples with $r \leqslant 1$, it would also hold for all the remaining couples with $r>1$.

Let $B_{k}:=\bar{B}_{r_{k}}\left(x_{0}\right)$ with $r_{k}=r 2^{-k}$. Since $Z$ is lower semicontinuous, we have

$$
Z\left(x_{0}\right)=\operatorname{supinf}_{k \geqslant 0} Z \text {. }
$$

Then, given $\varepsilon>0$ we must have $Z\left(x_{0}\right)-\varepsilon<\inf _{B_{k}} Z$ for every $k \geqslant k_{0}$ for a suitable integer $k_{0}$. Since $x \in B_{0}$, we clearly have $\inf _{B_{0}} Z \leqslant Z(x)$. For $\varepsilon=c r^{\beta} / 2$, we get

$$
\inf _{B_{k_{0}}} Z-\inf _{B_{0}} Z \geqslant \frac{1}{2} c r^{\beta} \text {. }
$$

Choose

$$
c^{\prime}=2^{\beta-1}\left(1-2^{-\beta}\right) c
$$

Since

$$
\inf _{B_{k_{0}}} Z-\inf _{B_{0}} Z=\sum_{k=1}^{k_{0}}\left(\inf _{B_{k}} Z-\inf _{B_{k-1}} Z\right),
$$

we cannot have

$$
\inf _{B_{k}} Z-\inf _{B_{k-1}} Z<c^{\prime} r_{k}^{\beta} \quad \text { for all } k=1, \ldots, k_{0},
$$

as then

$$
c<\frac{2^{1-\beta} c^{\prime}}{1-2^{-\beta}},
$$

contrary to 6.2. For a certain value of $k=k_{1}$ we must thus have

$$
\inf _{B_{k_{1}}} Z-\inf _{B_{k_{1}-1}} Z \geqslant c^{\prime} r_{k_{1}}^{\beta} \text {. }
$$

Let $x_{k_{1}-1}$ be a point which realizes the infimum of $Z$ on $B_{k_{1}-1}$. We now apply Corollary 3.18 with $X=B_{k_{1}}, y=x_{k_{1}-1}$, and the moved mass is that carried by fibers stopping on $X=B_{k_{1}}$. The difference of the values of the landscape function between $X=B_{k_{1}}$ and $y=x_{k_{1}-1}$ is then given by $c^{\prime} r_{k_{1}}^{\beta}$. Taking into account Corollary 5.4 since the irrigated measure is Ahlfors from below and $x_{0} \in \operatorname{spt} \mu$, inequality (3.8) of Corollary 3.18 becomes

$$
\alpha c^{\prime} r_{k}^{\beta} \leqslant C\left(r_{k}^{h}\right)^{\alpha-1} r_{k}=C r_{k}^{\beta} .
$$

Here $C$ is the constant of Corollary 5.4. This means that if we choose $c$, and therefore $c^{\prime}$, sufficiently large we get a contradiction.

Now, we reach the main theorem of the paper.

THEOREM 6.2 Let $\mu$ be the irrigated measure, which is supposed to be Ahlfors regular from below in dimension $h$. Let $Z$ be the landscape function associated to the optimal pattern $\chi$. Let $\beta:=$ $1+h(\alpha-1)$. Then

on $\bar{D}_{\chi}$ of the pattern $\chi$.

$$
|Z(x)-Z(y)| \leqslant c|x-y|^{\beta}
$$

Proof. The result simply follows from Lemma 6.1 and the equivalence stated in Theorem 4.5 . 


\section{Necessary conditions for the Hölder continuity of the landscape function}

The main result of this section is the following theorem. In Appendix A the reader will find the definitions of the various notions of measure dimension which play a role in this and the following sections.

ThEOREM 7.1 Let $\mu \in \mathcal{P}(\Omega)$. Let $\chi$ be an optimal pattern between $\delta_{S}$ and $\mu$. Suppose that the landscape function is Hölder continuous of exponent $\beta \leqslant \alpha$ (i.e. the decay exponent $h=$ $(1-\beta) /(1-\alpha)$ satisfies $h \geqslant 1)$. Then

$$
\operatorname{dim}_{M}(\mu) \leqslant h .
$$

From Theorem 7.1, we deduce a corollary which matches Theorem 6.2

DEFINITION 7.2 (Upper Ahlfors regular measure) A measure $\mu$ is Ahlfors regular from above in dimension $h$ if there exists $C_{A}>0$ such that

$$
\mu(B(x, r)) \leqslant C_{A} r^{h} .
$$

COROLlary 7.3 Under the same assumptions of Theorem 7.1. suppose also that $\mu$ is upper Ahlfors regular in dimension $h^{\prime}$. Then

$$
h^{\prime} \leqslant h=\frac{1-\beta}{1-\alpha}, \quad \text { i.e. } \quad \beta \leqslant 1+h^{\prime}(\alpha-1) .
$$

Proof of Corollary 7.3. Recall the following results from [DS2]:

- Theorem 1.1: $\operatorname{dim}_{\mathrm{c}}(\mu) \leqslant \max \left\{\operatorname{dim}_{\mathrm{M}}(\mu), 1\right\}$; recall that $\operatorname{dim}_{\mathrm{c}}(\mu)$ is the least Hausdorff dimension of a set on which $\mu$ is concentrated;

- Corollary 1.4: if $\mu$ is upper Ahlfors regular in $\operatorname{dimension} h^{\prime}$, then $\operatorname{dim}_{\mathrm{c}}(\mu) \geqslant h^{\prime}$.

We then have

$$
h^{\prime} \leqslant \operatorname{dim}_{\mathrm{c}}(\mu) \leqslant \max \left\{\operatorname{dim}_{\mathrm{M}}(\mu), 1\right\} \leqslant h,
$$

which completes the proof of the corollary.

COROLlaRY 7.4 If the irrigated measure is Ahlfors regular in dimension $h \geqslant 1$, the exponent given by Theorem 6.2 is the highest one and is independent of the source of irrigation.

Proof. Suppose that $\mu$ is Ahlfors regular from below in dimension $h_{1}$ and from above in dimension $h_{2}$. The exponents must satisfy $h_{2} \leqslant h_{1}$. By Theorem 6.2 the landscape function is Hölder with exponent $\beta_{1}=1+h_{1}(\alpha-1)$ (or less). If we add the hypothesis that $h_{2} \geqslant 1$, and apply Corollary 7.3 we find that the Hölder continuity exponent cannot exceed $\beta_{2}=1+h_{2}(\alpha-1) \geqslant \beta_{1}$. This shows that when $\mu$ is Ahlfors regular in dimension $h \geqslant 1$ (i.e., $h_{1}=h_{2}=h$, and $\beta_{1}=\beta_{2}=1+h(\alpha-1)$ ), the Hölder regularity given by Theorem 6.2 is the best possible and the regularity does not depend on the position of the source.

To be able to prove Theorem 7.1 we need some preliminary results and notation.

REMARK 7.5 If the landscape function is Hölder continuous of exponent $\beta$, then the length of the fibers is bounded by $\|\bar{Z}\|_{\mathrm{C}^{\beta}}(\operatorname{diam}(\{S\} \cup \operatorname{spt} \mu))^{\beta}$ on the support of the pattern $D_{\chi}$. 
Indeed, if $p$ is a fiber, then its length is given by $\int_{I}|\dot{\chi}(p, t)| \mathrm{d} t$. Thanks to the Hölder continuity of the landscape function we can estimate the length as

$$
\int_{I}|\dot{\chi}(p, t)| \mathrm{d} t \leqslant \bar{Z}(\chi(p, b))-\bar{Z}(S) \leqslant\|\bar{Z}\|_{\mathrm{C}^{\beta}}|S-\chi(p, b)|^{\beta} \leqslant C(\operatorname{diam}(\{S\} \cup \operatorname{spt} \mu))^{\beta} .
$$

Since the left hand-side is the length of the fiber, the statement is proved.

Given $p \in \Omega$ and a point $x$ define $t_{p}(x)$ as

$$
t_{p}(x):=\inf \{t \in I: \chi(p, t)=x\} .
$$

Coherently, $t_{p}(x)=b:=\sup I$ if the fiber $p$ does not pass through $x$. Consider the function $l$ defined on the image of an optimal pattern $\chi$ which associates to every $x$ the supremum of the distance along the fiber $\chi_{p}$ from $x$ to the terminal point of the fiber, given by

$$
l(x)=\operatorname{ess} \sup l_{p}(x),
$$

where

$$
l_{p}(x):=\int_{t_{p}(x)}^{b}\left|\dot{\chi}_{p}(s)\right| \mathrm{d} s
$$

(if $Z$ is Hölder continuous, $l_{p}(x)<+\infty$ for a.e. $p \in \Omega$ by Remark 7.5 , and the essential supremum is taken over the particles $p$ whose equivalence class at the time when it passes through $x$ is of positive measure, i.e. $\left|[p]_{t_{p}(x)}\right|>0$.

With this notation, the implication (i) $\Rightarrow$ (iii) in Theorem 4.3 can be restated as: if $Z$ is Hölder continuous of exponent $\beta$, then

$$
m_{\chi}(x) \geqslant C l(x)^{h}
$$

for some constant $C$ and $h=(1-\beta) /(1-\alpha)$.

Let us recall a definition from [MS2].

Definition 7.6 (Flow ordering) Consider an optimal pattern $\chi$. Let $x, y \in \mathbb{R}^{N}$. We say that $x$ precedes $y$ in the flow order if there exists $A \subseteq \Omega$ with $\mu_{\Omega}(A)>0$ such that for all $p \in A$ we have $c(p)<+\infty$ and $t_{x} \leqslant t_{y}$, where $\chi_{p}\left(t_{x}\right)=x, \chi_{p}\left(t_{y}\right)=y$. In this case we write $x \leqslant y$. Note this is a partial ordering.

Lemma 7.7 Consider an optimal pattern $\chi$. Suppose that $x \leqslant y$. For a.e. $p \in \Omega$,

$$
l(y)-l(x) \leqslant-d(x, y) .
$$

As a consequence, $l$ is decreasing with respect to $\leqslant$.

Proof. If $x, y \in \chi_{p}(I)$, we must have by definition

$$
l_{p}(x)-l_{p}(y)=d(x, y) .
$$

Formula 7.1 implies that for every $\varepsilon>0$, there exists a set $P$ of fibers with a positive measure passing through $y$ such that if $q_{\varepsilon} \in P$ then

$$
l(y) \leqslant l_{q_{\varepsilon}}(y)+\varepsilon .
$$


Since $\chi$ is a simple pattern, $x \in \chi_{q_{\varepsilon}}$. We then have, for some $q_{\varepsilon}$,

$$
l(x) \geqslant l_{q_{\varepsilon}}(x)=l_{q_{\varepsilon}}(y)+d_{q_{\varepsilon}}(x, y)=l_{q_{\varepsilon}}(y)+d(x, y) \geqslant l(y)-\varepsilon+d(x, y) .
$$

Since $\varepsilon>0$ can be chosen arbitrarily small, the lemma is proved.

LEMMA 7.8 Consider an optimal pattern $\chi$. Suppose that the landscape function is Hölder continuous of exponent $\beta$. Then $l \circ \chi_{p}$ is upper semicontinuous for a.e. $p \in \Omega$.

Proof. $l \circ \chi_{p}$ is decreasing by Lemma 7.7, so we just need to prove the left continuity. Given $t_{0} \in I$ and any increasing sequence $t_{n} \rightarrow t_{0}$ we will prove that

$$
c<\lim _{n \rightarrow+\infty} l\left(\chi_{p}\left(t_{n}\right)\right) \Rightarrow c \leqslant l\left(\chi_{p}\left(t_{0}\right)\right)
$$

This will imply $\lim _{n} l\left(\chi_{p}\left(t_{n}\right)\right)=l\left(\chi_{p}\left(t_{0}\right)\right)$. Note that, in principle, we could have $c>l\left(\chi_{p}\left(t_{0}\right)\right)$ if the fibers passing through $\chi_{p}\left(t_{n}\right)$ and almost realizing the value of $l\left(\chi_{p}\left(t_{n}\right)\right)$ would not also pass through $\chi_{p}\left(t_{0}\right)$. In the following we will rule out this occurrence, showing that there must be a set of fibers of positive measure passing through $\chi_{p}\left(t_{0}\right)$ and of "residual length" at least $c$. Let

$$
A_{n}=\left\{q \in[p]_{t_{n}}: l_{q}\left(\chi_{p}\left(t_{n}\right)\right) \geqslant c\right\} .
$$

We have $A_{n+1} \subseteq A_{n}$, since $[p]_{t_{n+1}} \subseteq[p]_{t_{n}}$ and $l_{q}\left(\chi_{p}\left(t_{n}\right)\right) \geqslant l_{q}\left(\chi_{p}\left(t_{n+1}\right)\right)$. Let $c^{\prime}$ be any number such that

$$
c<c^{\prime}<\lim _{n \rightarrow+\infty} l\left(\chi_{p}\left(t_{n}\right)\right)
$$

Since $l\left(\chi_{p}\left(t_{n}\right)\right)>c^{\prime}$, we can find $p_{n} \in[p]_{t_{n}}$ (with $[p]_{t_{n}}$ of positive measure) such that $l_{p_{n}}\left(\chi_{p}\left(t_{n}\right)\right)>c^{\prime}$. Then, if we take $\tau_{n}>t_{n}$ such that $d\left(\chi_{p_{n}}\left(t_{n}\right), \chi_{p_{n}}\left(\tau_{n}\right)\right)=c$, we have

$$
l_{p_{n}}\left(\chi_{p_{n}}\left(\tau_{n}\right)\right)>c^{\prime}-c .
$$

Therefore, from inequality 7.3 ,

$$
\left|\left[p_{n}\right]_{\tau_{n}}\right| \geqslant C\left(c^{\prime}-c\right)^{h}
$$

where, as usual, $h=(1-\beta) /(1-\alpha)$. Obviously, $\left[p_{n}\right]_{\tau_{n}} \subseteq A_{n}$, so

$$
\mu_{\Omega}\left(A_{n}\right) \geqslant C\left(c^{\prime}-c\right)^{h} \text {. }
$$

Let

$$
A=\bigcap_{n>0} A_{n}
$$

Then

$$
\mu_{\Omega}(A) \geqslant C\left(c^{\prime}-c\right)^{h}>0 .
$$

Every fiber $q \in A$ passes through $\chi_{p}\left(t_{0}\right)$ (thanks to the continuity of $\left.\chi_{p}\right)$, and from $l_{q}\left(\chi_{p}\left(t_{n}\right)\right) \geqslant c$ it follows that $l_{q}\left(\chi_{p}\left(t_{0}\right)\right) \geqslant c$. Consequently, $l\left(\chi_{p}\left(t_{0}\right)\right) \geqslant c$.

We now go on with a key proposition in the proof of Theorem 7.1. This is a kind of "intermediate value theorem" for $l$ (in spite of the fact that $l$ is not continuous).

Proposition 7.9 Suppose that $l$ is the function associated to an optimal pattern $\chi$. Let $l_{1} \leqslant l\left(x_{1}\right)$. Then there exists $x_{2} \geqslant x_{1}$ such that $l\left(x_{2}\right)=l_{1}$. 
Proof. Let $l_{1}>0$, otherwise we just choose a terminal point. Consider the following minimization problem:

$$
m=\inf \left\{l(y): y \geqslant x_{1}, l(y) \geqslant l_{1}\right\} .
$$

Let $y_{n}$ be a minimizing sequence and suppose $l\left(y_{n}\right) \geqslant l\left(y_{n+1}\right)$ and $l\left(y_{n}\right) \rightarrow m \geqslant l_{1}$. Let

$$
P_{n}=\left\{p \in \Omega: y_{n} \in \chi_{p}(I)\right\} .
$$

Because of our hypothesis on the Hölder continuity of the landscape function, we deduce from inequality $(7.3)$ that

$$
\mu_{\Omega}\left(P_{n}\right) \geqslant C l_{1}^{h}
$$

Let

$$
P=\limsup _{n \rightarrow+\infty} P_{n}=\bigcap_{n \geqslant 0} \bigcup_{k \geqslant n} P_{k}
$$

By the Fatou Lemma,

$$
\mu_{\Omega}(P) \geqslant \limsup _{n \rightarrow+\infty} \mu_{\Omega}\left(P_{n}\right) \geqslant C l_{1}^{h} .
$$

By the definition of $P$, if $p \in P$ then for some increasing sequences $n_{k}$ and $t_{k}$ we must have $\chi\left(p, t_{k}\right)=y_{n_{k}}$. Finally, $l\left(y_{n_{k}}\right) \geqslant l_{1}$ and $t_{k} \rightarrow t_{\infty}$ (since $y_{n}$ is a minimizing sequence on which $l$ is decreasing).

Let $y_{\min }:=\chi\left(p, t_{\infty}\right)$. Then $y_{n_{k}} \rightarrow y_{\min }$ and by Lemma 7.8

$$
l\left(y_{\min }\right) \geqslant \limsup _{k \rightarrow+\infty} l\left(y_{n_{k}}\right)=\lim _{k \rightarrow+\infty} l\left(y_{n_{k}}\right) \geqslant l_{1}
$$

so $y_{\min }$ solves (7.4), since for all $k$ we have $l\left(y_{\min }\right) \leqslant l\left(y_{n_{k}}\right)\left(y_{n_{k}}\right.$ precedes $y_{\min }$ and $l$ is monotone on the fiber as stated in Lemma 7.7p.

If we now set $x_{2}=y_{\min }$, we just need to prove that $l\left(y_{\min }\right)=l_{1}$. Suppose, on the contrary, that $l\left(y_{\min }\right)>l_{1}$. Then, for some fiber $p_{1}, l_{p_{1}}\left(y_{\min }\right)>l_{1}$. Hence for some $\varepsilon_{1}$ we would find a point $\bar{y}=\chi\left(p_{1}, t_{\infty}+\varepsilon_{1}\right) \geqslant x_{1}$ with $l_{p_{1}}(\bar{y})>l_{1}$ and, by Lemma 7.7 $l(\bar{y})<l\left(y_{\min }\right)$. Then $y_{\text {min }}$ would not be the minimizer of the problem stated in (7.4), since $\bar{y}$ would satisfy the constraints and $l(\bar{y})<l\left(y_{\min }\right)$.

Proof of Theorem 7.1 Consider the following construction. We start from a terminal point $x_{0}$ and go back along its fiber $p$ a distance $\delta$. We reach a point $x_{1}$ where $l\left(x_{1}\right) \geqslant l_{p}\left(x_{1}\right)=\delta$. Let $x_{2} \geqslant x_{1}$ be the point in Proposition 7.9 such that $l\left(x_{2}\right)=k \delta$ for the maximum positive integer $k$ possible. Since $k$ is maximum, the Euclidean distance between $x_{0}, x_{2}$ is at most $2 \delta$ by Lemma 7.7. Note that the maximum integer $k(\delta)$ obtained in this way does not exceed $\delta^{-1} C d^{\beta}$ (where $C$ is the Hölder constant of the landscape function and $d=\operatorname{diam}(\{S\} \cup \operatorname{spt} \mu)$ ). Indeed, by Remark7.5 we have

$$
k(\delta) \delta=l\left(x_{2}\right) \leqslant C d^{\beta} .
$$

Let $S(\delta)$ the set of points obtained like $x_{2}$ from a terminal point $x_{0}$, and $v$ the measure which realizes the minimum of the $\infty$-Wasserstein distance between the irrigated measure $\mu$ and the set of probability measures supported on $S(\delta)$. The distance of each of the selected points to the support of the final measure is at most $2 \delta$, so that $w_{\infty}(\mu, \nu) \leqslant 2 \delta$. 
Given $k$, the number of points of $S(\delta)$ such that $l(x)=k \delta$ can be estimated by $C^{1 /(1-\alpha)} \delta^{-h} k^{-h}$ : indeed, these points cannot be on a flow line by the way they are chosen, so their number $N_{k}$ must satisfy

$$
C^{1 /(\alpha-1)} N_{k}(k \delta)^{h} \leqslant 1,
$$

since by 7.3 each point carries a mass at least $C^{1 /(\alpha-1)}(k \delta)^{h}$ and their total mass must be less than 1 .

Hence, for the total number of points $N(\delta)$ we have the following estimate:

$$
N(\delta)=\sum_{k=1}^{k(\delta)} N_{k} \leqslant C^{1 /(1-\alpha)} \delta^{-h}\left[1+\int_{1}^{\delta^{-1} C d^{\beta}} x^{-h} \mathrm{~d} x\right]
$$

In the case $h>1$,

$$
\int_{1}^{\delta^{-1} C d^{\beta}} x^{-h} \mathrm{~d} x=-\frac{\left[\delta C^{-1} d^{-\beta}\right]^{h-1}-1}{h-1} \leqslant-\log \left(\delta C^{-1} d^{-\beta}\right) .
$$

The last inequality follows from the convexity of the exponential function. In the case $h=1$,

$$
\int_{1}^{\delta^{-1} C d^{\beta}} x^{-1} \mathrm{~d} x=-\log \left(\delta C^{-1} d^{-\beta}\right) .
$$

In any case, we can estimate

$$
N(\delta) \leqslant C^{1 /(1-\alpha)} \delta^{-h}\left(1-\log \left(\delta C^{-1} d^{-\beta}\right)\right)=: \delta^{-h} f(\delta)
$$

where $f(\delta)$ grows as $-\log \delta$ as $\delta \rightarrow 0^{+}$. Now, given $n$ choose the unique $\delta$ (the uniqueness is true only for $\delta$ small) such that $n=\delta^{-h} f(\delta)$. For such a choice of $\delta$,

$$
w_{\infty}\left(\mu, D_{n}\right) \leqslant w_{\infty}\left(\mu, D_{N(\delta)}\right) \leqslant 2 \delta
$$

We then easily have

$$
\operatorname{dim}_{\mathrm{res}}^{\infty}(\mu)=\limsup _{n \rightarrow+\infty}\left(\frac{\log w_{\infty}\left(\mu, D_{n}\right)}{-\log n}\right)^{-1} \leqslant \limsup _{\delta \rightarrow 0^{+}}\left(\frac{\log (2 \delta)}{-\log \left(\delta^{-h} f(\delta)\right)}\right)^{-1}=h .
$$

The statement of the theorem now follows from the following result of [DS2, Proposition 5.4]: $\operatorname{dim}_{M}(\mu)=\operatorname{dim}_{\text {res }}^{\infty}(\mu)$.

REMARK 7.10 Note that in the previous proof, in the case $h>1, N(\delta)$ can be estimated by

$$
N(\delta)=\sum_{k=1}^{k(\delta)} N_{k} \leqslant C^{1 /(1-\alpha)} \delta^{-h}\left[1+\int_{1}^{+\infty} x^{-h} \mathrm{~d} x\right]
$$

Since $N(\delta)$ does not depend on $k(\delta)$ and, consequently, on the size $d$ of the diameter of the convex hull of the supports of the initial and final measure, the irrigated measure does not need to be compactly supported. Of course, this is not the case when $h=1$. 


\section{Counter-examples}

We now provide some counter-examples which show the role of the hypotheses assumed in this paper.

Consider a probability measure $\mu \in \mathcal{P}(\mathbb{R})$. Its distribution function is $F(x)=\mu((-\infty, x))$ (this definition is slightly different from the usual one). Recall that $F$ is a non-decreasing, left-continuous (hence, lower semicontinuous) function. Moreover, $0 \leqslant F(x) \leqslant 1$ and

$$
\lim _{x \rightarrow-\infty} F(x)=0, \quad \lim _{x \rightarrow+\infty} F(x)=1 .
$$

Set

$$
J\left(x_{0}\right):=\lim _{x \rightarrow x_{0}^{+}} F(x)-F\left(x_{0}\right)=\mu\left(\left\{x_{0}\right\}\right)
$$

Given $y \in[0,1]$, the set

$$
\left.\left.F^{-1}(]-\infty, y\right]\right)=\{x \in \mathbb{R}: F(x) \leqslant y\}
$$

is a closed interval of the form $]-\infty, \alpha]$. We then set for $y \in[0,1[$, taking advantage of the lower semicontinuity of $F$,

$$
G(y):=\max \{x \in \mathbb{R}: F(x) \leqslant y\} .
$$

$G$ is the so-called quantile function of $F$. Since

$$
F^{-1}((-\infty, y])=(-\infty, G(y)]
$$

we have by construction

$$
F(x) \leqslant y \Leftrightarrow x \leqslant G(y) .
$$

$G$ is non-decreasing and right-continuous (hence, upper semicontinuous). Of course, if $F$ is one-toone, then $G$ is just $F^{-1}$. Note also that in general this may not happen because $\mu$ can be null on some interval.

Suppose now that we are given a measure $\mu$ such that spt $\mu \subseteq[0,1]$ and consider the problem of the irrigation of $\mu$ from $S=0$ (in the following we will always consider the irrigation from $S=0$, unless otherwise stated). The optimal pattern is given on $\Omega=[0,1]$ by

$$
\chi(p, t)=\min \{t, G(p)\},
$$

since the support must be convex and the no-loop condition must hold, and the multiplicity is given by

$$
m(x)=1-F(x)=\mu([x, 1]) .
$$

The landscape function is then given by

$$
Z(x)=\int_{0}^{x} m(t)^{\alpha-1} \mathrm{~d} t=\int_{0}^{x}(1-F(t))^{\alpha-1} \mathrm{~d} t .
$$

We have seen in Corollary 7.4 that when $\mu$ is Ahlfors regular in dimension $h$, the best Hölder exponent of the landscape function is $1+h(\alpha-1)$ and it does not depend on how we choose the source $S$. The same is not true if the irrigated measure is only Ahlfors regular from below, as the following example shows. 
EXAMPLE 8.1 When the measure is Ahlfors regular from below in dimension $h$, the regularity of the landscape function may depend on the location of the source $S$ and may assume both the lowest best possible value $1+h(\alpha-1)$ (given by Theorem 6.2) and the highest best possible value 1 . Consider the measure $\mu \in \mathcal{P}([0,1])$ given by

$$
\mu=\frac{1}{2} \delta_{0}+\frac{1}{2} f \mathcal{L}_{\mid[0,1]}^{1}
$$

where $f(x)=h(1-x)^{h-1}$ with $h>1$. It is easy to check that $\mu$ is Ahlfors regular from below in dimension $h$ (but not from above). The distribution function of $\mu$ is given by

$$
F(x)=1-\frac{1}{2}(1-x)^{h} .
$$

Suppose now that $\alpha>1-1 / h$. By formula 8.1 we have

$$
Z(x)=\int_{0}^{x} \frac{1}{2^{\alpha-1}}(1-t)^{h(\alpha-1)} \mathrm{d} t=\frac{1}{2^{\alpha-1} \beta}\left(1-(1-x)^{\beta}\right),
$$

where $\beta=1+h(\alpha-1)$. Hence $Z$ is then Hölder continuous with the exponent given by Theorem 6.2. Note that in this case $x=1$ is a terminal point. The same regularity holds for $S<1$.

On the other hand, if we irrigate the same measure from a point $S \geqslant 1$, the mass function in $0 \leqslant x \leqslant 1$ is given by

$$
m(x)=1-\frac{1}{2}(1-x)^{h} \geqslant \frac{1}{2},
$$

while $m(x)=0$ if $x \geqslant 1$. The landscape function is given by

$$
Z_{1}(x)=\int_{0}^{x} m(x)^{\alpha-1} \mathrm{~d} t+S-1,
$$

which is Lipschitz continuous, the best possible regularity of the landscape function.

An analogous construction leads to the following remark.

EXAMPLE 8.2 (A counterexample to the conclusion of Corollary 7.3 if we drop the hypothesis $h \geqslant 1$ ) An estimate analogous to inequality (7.5) in the case $h<1$ actually provides only $\operatorname{dim}_{\mathrm{M}}(\mu) \leqslant 1$, which gives $h^{\prime} \leqslant \operatorname{dim}_{\mathrm{c}}(\mu) \leqslant \min \left\{\operatorname{dim}_{\mathrm{M}}(\mu), 1\right\}=1$ since $\operatorname{dim}_{\mathrm{M}}(\mu) \leqslant h<1$. Actually, this is in the nature of things. Indeed, consider the irrigation from $S=0$ of the measure

$$
\mu=\frac{1}{2} \mathcal{L}_{\mid[0,1]}^{1}+\frac{1}{2} \delta_{1}
$$

Since the multiplicity is bounded from below, the landscape function is Lipschitz continuous $(h=0)$, but the measure has Minkowski dimension $h^{\prime}=1$.

In general, without assumptions on the irrigated measure, the landscape function may be no more than lower semicontinuous. This may happen in dimension greater than one (as the next example shows). In the 1-dimensional case, the continuity is guaranteed by the following proposition.

PROPOSITION 8.3 In the 1-dimensional case, the landscape function is continuous. Moreover, it is locally Lipschitz continuous in the set where it is finite. 
Proof. Let $[a, b]$ be the convex hull of the support of the irrigated measure and the source of irrigation. Without any restriction, let $S=0$ be the source of irrigation. The landscape function is then defined on $[a, b]$. Suppose that $a \geqslant 0$. Since the function $(1-F(x))^{\alpha-1}$ is non-decreasing, it is bounded in any interval of the type $[0, b-\varepsilon]$, so that $Z \in \operatorname{Lip}_{\text {loc }}([0, b-\varepsilon])$ and the proposition is proved.

If $a<0$, the optimal pattern is built up merging the optimal pattern irrigating $\mu_{\mid[0, b]}$ and $\mu_{\mid[a, 0]}$. This proves the continuity and Lipschitz continuity separately, which ultimately gives the continuity of the landscape function on $[a, b]$ and its locally Lipschitz continuity on $] a, b[$.

The landscape function remains continuous even if it takes the value $+\infty$. It is easy to see that this may happen for an irrigable measure, as shown, for instance, in the following example.

REMARK 8.4 Note that we may have $Z(b)=+\infty$ (but $\mu$ is nevertheless irrigable). This is the case for a measure $\mu$ whose density is given by

$$
f(x)=h(1-x)^{h-1} \mathcal{L}_{\mid[0,1]}^{1} .
$$

In this case $b=1$ and $1-F(x)=(1-x)^{h}$. If we take $h(1-\alpha)>1$, for $x<1$ we have

$$
Z(x)=\int_{0}^{x}(1-F(t))^{\alpha-1} \mathrm{~d} t=\frac{1}{1+h(\alpha-1)}-\frac{(1-x)^{1+h(\alpha-1)}}{1+h(\alpha-1)},
$$

while $Z(1)=+\infty$. Nevertheless, $\mu$ is irrigable since in the 1-dimensional case every measure is irrigable for $0<\alpha \leqslant 1$.

EXAMPLE 8.5 The landscape function is, in general, not continuous in dimension $N \geqslant 2$. Let $\alpha \in[0,1[$ and let the source be $S=(0,0)$.

(i) Consider any sequence $\left\{x_{n}\right\}_{n} \geqslant 1$ of points in $\mathbb{R}^{2}$ such that:

- $x_{n} \rightarrow S$ as $n \rightarrow+\infty$;

- for every $n \geqslant 1$, the point $x_{n+1}$ is at a positive distance from the (closed) convex envelope $C_{n}$ of $S, x_{1}, \ldots, x_{n}$.

For example, the sequence given, for $n \geqslant 1$, by

$$
x_{n}=\frac{1}{n}\left(\cos \left(\frac{\pi}{2 n}\right), \sin \left(\frac{\pi}{2 n}\right)\right)
$$

would fit these requirements.

(ii) Consider now the following recurrent construction. Suppose that, given $n \geqslant 1$, we have defined a measure

$$
\mu_{n}=\sum_{k=1}^{n} a_{k} \delta_{x_{k}} \quad \text { with } \quad \sum_{k=1}^{n} a_{k}=1 .
$$

Note that this condition determines $\mu_{1}$ (which turns out to be $\delta_{x_{1}}$ ). Define

$$
\mu_{n}^{a}=a v+\left(a_{n}-a\right) \delta_{x_{n}}+\sum_{k=1}^{n-1} a_{k} \delta_{x_{k}},
$$

where $v$ is any probability measure supported in the closure of the set $\left\{x_{k}: k>n\right\}$. Since $\mu_{n}^{a} \rightarrow \mu_{n}$ as $a \rightarrow 0^{+}, \chi_{n}^{a}(p, \cdot) \rightarrow \chi_{n}(p, \cdot)$ uniformly for a.e. $p \in \Omega$ by the Skorokhod Theorem. 
(iii) Fix a radius $r_{n+1}$ such that $B_{r_{n+1}}\left(x_{n+1}\right)$ and $C_{n}$ are a positive distance apart. We now prove that for every $\varepsilon>0$ there exists $\delta_{n}(\varepsilon)>0$ such that, whenever $a<\delta_{n}(\varepsilon)$, the measure of the set

$$
P_{n}^{a}=\left\{p \in \Omega: \chi_{n}(p, I) \cap B_{r_{n+1}}\left(x_{n+1}\right) \neq \emptyset\right\}
$$

is less than $\varepsilon$. Indeed, suppose on the contrary that for some $\varepsilon>0$ there exists a sequence $a_{k} \rightarrow 0$ such that $\mu_{\Omega}\left(P_{n}^{a_{k}}\right) \geqslant \varepsilon$. Then

$$
\mu_{\Omega}\left(\limsup _{k \rightarrow+\infty} P_{n}^{a_{k}}\right) \geqslant \limsup _{k \rightarrow+\infty} \mu_{\Omega}\left(P_{n}^{a_{k}}\right) \geqslant \varepsilon
$$

which contradicts the a.e. uniform convergence given by the Skorokhod Theorem (the previous step).

(iv) Fix now $\varepsilon_{n+1}$ such that $\varepsilon_{n+1}^{\alpha-1} r_{n+1}>n+1$ and $a_{n+1}<\min \left\{a_{n}, \delta_{n}\left(\varepsilon_{n+1}\right)\right\}$ such that $P_{n}^{a_{n+1}}$ has measure less than $\varepsilon_{n+1}$.

(v) Consider now an optimal pattern $\chi$ irrigating the measure $\mu$ built in this way. This pattern is irrigable if $\alpha>1 / 2$ (note that we could also require $\sum_{n} a_{n}^{\alpha}\left|x_{n}\right|<+\infty$, so that the measure would also be irrigable for $\alpha \leqslant 1 / 2)$. By construction, $Z\left(x_{n}\right) \geqslant \varepsilon_{n}^{\alpha-1} r_{n}>n$ since a mass of at most $\varepsilon_{n}$ has to cover a distance at least $r_{n}$ to reach $x_{n}$. Then

$$
\lim _{n \rightarrow+\infty} Z\left(x_{n}\right)=+\infty>0=Z(S)
$$

The landscape function is thus lower semicontinuous, but not continuous at $S$.

\section{A. Dimensions}

In this appendix we recall the definitions and main properties of the dimensions used in the paper. For the details we refer to [DS2] or [M].

\section{A.1 Hausdorff dimension}

Given a set $A$, its Hausdorff dimension is defined by

$$
\operatorname{dim}_{\mathcal{H}}(A):=\inf \left\{\alpha \geqslant 0: \mathcal{H}^{\alpha}(A)=0\right\}
$$

DEFINITION A.1 (Hausdorff concentration dimension) Given a Borel measure $\mu$, we define the Hausdorff concentration dimension of $\mu$ as

$$
\operatorname{dim}_{\mathrm{c}}(\mu)=\inf \left\{\operatorname{dim}_{\mathcal{H}}(B): \mu\left(B^{c}\right)=0\right\}
$$

Proposition A.2 If a Borel measure $\mu$ is upper Ahlfors regular in dimension $h^{\prime}$, then $\operatorname{dim}_{\mathrm{c}}(\mu) \geqslant h^{\prime}$.

Proof. See Corollary 1.4 of [DS2]. 


\section{A.2 Minkowski dimension}

Minkowski dimension can be defined in several ways. Let $A$ be a bounded subset of $\mathbb{R}^{N}$. Define $N(A, \varepsilon)$ as

$$
N(A, \varepsilon):=\min \left\{k \in \mathbb{N}: A \subseteq \bigcup_{i=1}^{k} B_{\varepsilon}\left(x_{i}\right), x_{i} \in \mathbb{R}^{N}\right\} .
$$

$N(A, \varepsilon)$ is the least number of balls of radius $\varepsilon$ whose union covers $A$.

Definition A.3 (Minkowski dimension) The Minkowski dimension of a set $A$ is defined as

$$
\operatorname{dim}_{\mathrm{M}}(A):=\inf \left\{\alpha \geqslant 0: \limsup _{\varepsilon \rightarrow 0^{+}} N(A, \varepsilon) \varepsilon^{\alpha}=0\right\} .
$$

Definition as a power of $\varepsilon^{-1}$. It is easy to see that Minkowski dimension is also given by

$$
\operatorname{dim}_{\mathrm{M}}(A)=\limsup _{\varepsilon \rightarrow 0^{+}} \frac{\log N(A, \varepsilon)}{-\log \varepsilon}=\limsup _{\varepsilon \rightarrow 0^{+}} \log _{1 / \varepsilon} N(A, \varepsilon) .
$$

The Minkowski dimension measures how fast $N(A, \varepsilon)$ grows as $\varepsilon \rightarrow 0^{+}$in terms of a power of $\varepsilon^{-1}$.

Definition via the Minkowski content. Another equivalent definition is

$$
\operatorname{dim}_{\mathrm{M}}(A):=N+\limsup _{\varepsilon \rightarrow 0^{+}} \frac{\log \mathcal{L}^{N}\left(A_{\varepsilon}\right)}{-\log \varepsilon},
$$

where $A_{\varepsilon}$ is the closed $\varepsilon$-neighborhood of $A$ given by

$$
A_{\varepsilon}:=\left\{x \in \mathbb{R}^{N}: d(x, A) \leqslant \varepsilon\right\} .
$$

Definition as box counting dimension. There is one more way to define Minkowski dimension. Recall that a dyadic cube of order $m$ in $\mathbb{R}^{N}$ is a Cartesian product of $N$ intervals of the kind $\left[k 2^{-m},(k+1) 2^{-m}\right.$ [ for $k \in \mathbb{Z}, m \in \mathbb{N}$. For every given $m$, the dyadic cubes of order $m$ are a disjoint cover of $\mathbb{R}^{N}$. Let $Q(A, m)$ be the cardinality of dyadic cubes of order $m$ which meet $A$. The Minkowski dimension of $A$ is given by

$$
\operatorname{dim}_{\mathrm{M}}(A)=\limsup _{\varepsilon \rightarrow 0^{+}} \frac{\log Q(A, m)}{m \log 2} .
$$

Proposition A.4 For every set $A$, we have

$$
\operatorname{dim}_{\mathcal{H}}(A) \leqslant \operatorname{dim}_{\mathrm{M}}(A)
$$

Definition A.5 (Minkowski dimension) The Minkowski dimension of a measure $\mu$ is given by the infimum of the Minkowski dimensions of the sets $B$ on which $\mu$ is concentrated (or equivalently of the support of $\mu$ ). It is denoted by $\operatorname{dim}_{M}(\mu)$.

Proposition A.6 Let $\mu \in \mathcal{P}\left(\mathbb{R}^{N}\right)$. Then

$$
\operatorname{dim}_{c}(\mu) \leqslant \max \left\{\operatorname{dim}_{M}(\mu), 1\right\} .
$$

Proof. See Theorem 1.1 of [DS2]. 


\section{A.3 Resolution dimension}

The resolution dimension was introduced by Devillanova and Solimini in [DS2], to which we refer for the proof of the statements given. Let $\mu \in \mathcal{P}(\Omega)$. Consider the set $D_{n}$ of discrete measures $v$ with \# $(\operatorname{spt} v) \leqslant n$ and the minimization problem

$$
w_{p}\left(\mu, D_{n}\right):=\min _{v \in D_{n}} w_{p}(\mu, v) .
$$

Definition A.7 (Resolution dimension) Let $\mu \in \mathcal{P}\left(\mathbb{R}^{N}\right)$ and $p \in[1,+\infty]$. Then the resolution dimension of $\mu$ of index $p$ is given by

$$
\operatorname{dim}_{\mathrm{res}}^{p}(\mu):=-\left(\limsup _{n \rightarrow+\infty} \frac{\log w_{p}\left(\mu, D_{n}\right)}{\log n}\right)^{-1} .
$$

The next proposition is contained in [DS2].

Proposition A.8 For all probability measures $\mu$ we have

$$
\operatorname{dim}_{\mathcal{H}}(\mu) \leqslant \operatorname{dim}_{\text {res }}^{p}(\mu) \leqslant \operatorname{dim}_{\mathrm{M}}(\mu) .
$$

Moreover for $p=+\infty$ the resolution dimension coincides with the Minkowski dimension.

\section{B. Measurability facts}

Recall that in the model considered, a pattern $\chi: \Omega \times I \rightarrow \mathbb{R}^{N}$ is a Carathéodory function. This means that:

- for all $t \in I$ the function $p \mapsto \chi_{t}(p):=\chi(p, t)$ is measurable;

- for $\mu_{\Omega}$-a.e. $p \in \Omega$ the function $t \mapsto \chi_{p}(t):=\chi(p, t)$ is continuous.

DEFINITION B. 1 (Fibers passing through a point $x$ ) Given $x \in \mathbb{R}^{N}$, consider the set $T(x)$ defined by

$$
T(x):=\{p \in \Omega: x \in \chi(p, I)\} .
$$

Equivalently, $T(x)$ is the set of fibers $p \in \Omega$ such that for some $t \in I$ we have $\chi(p, t)=x$, i.e. the fibers passing through $x$. Hence,

$$
T(x)=\bigcup_{t \in I} \chi_{t}^{-1}(\{x\}) .
$$

REMARK B.2 $T(x)$ is nothing other than $T(\{x\})$ of Definition 3.13

Proposition B.3 $T(x)$ is a measurable set.

Proof. The set $\chi_{t}^{-1}(\{x\})$ is measurable for all $t \in I$. Of course, this is not sufficient to prove the measurability since equation (B1) does not define $T(x)$ as a countable union. Let $I_{m}$ be an increasing sequence of compact intervals whose union is $I$ (if $I$ is itself compact, choose $I_{m}=I$ for all $m$ ). Define

$$
T_{m, n}(x)=\bigcup_{t \in I_{m}} \chi_{t}^{-1}\left(B_{1 / n}(x)\right), \quad T_{m}(x)=\bigcup_{t \in I_{m}} \chi_{t}^{-1}(\{x\}) .
$$

We have

$$
T_{m}(x)=\bigcap_{n>0} T_{m, n}(x), \quad T(x)=\bigcup_{m>0} T_{m}(x) .
$$


First, we prove that $T_{m, n}(x)$ is a measurable set. We have

$$
T_{m, n}(x)=\bigcup_{t \in I_{m} \cap \mathbb{Q}} \chi_{t}^{-1}\left(B_{1 / n}(x)\right) .
$$

Indeed, if $\bar{p} \in T_{m, n}(x)$, then $\chi(\bar{p}, \bar{t}) \in B_{1 / n}(x)$, so, by the continuity of $\chi$ with respect to the variable $t, \chi(\bar{p}, t) \in B_{1 / n}(x)$ for $t$ in a suitable open interval containing $\bar{t}$. In particular, $\chi(\bar{p}, \tilde{t}) \in$ $B_{1 / n}(x)$ for some $\tilde{t} \in \mathbb{Q}$. Since in equation (B3), $T_{m, n}(x)$ is a countable union of measurable sets, it is measurable.

We now prove that the first equality in $\mathrm{B} 2$ holds, the second being straighforward. Since $T_{m}(x) \subseteq T_{m, n}(x)$ for every $n$,

$$
T_{m}(x) \subseteq \bigcap_{n>0} T_{m, n}(x) .
$$

Now, suppose that $\bar{p} \in \bigcap_{n} T_{m, n}(x)$. For every $n>0$ there must be $t_{n} \in I_{m}$ such that $\chi\left(\bar{p}, t_{n}\right) \in$ $B_{1 / n}(x)$. Since $I_{m}$ is compact, up to a subsequence $t_{n} \rightarrow \bar{t} \in I_{m}$. Clearly, $\chi(\bar{p}, \bar{t})=x$, that is $\bar{p} \in B_{1 / n}(x)$.

This result can be easily generalized to a general closed set $X$.

Proposition B.4 If $X$ is a closed subset of $\mathbb{R}^{N}$, then $T(X)$ is a measurable set.

Proof. Just replace $B_{1 / n}(x)$ by the $1 / n$-neighborhood of $X$ in the proof of Proposition B.3

\begin{tabular}{|c|c|}
\hline $\mathbb{R}^{N}:$ & the Euclidean $N$-dimensional space \\
\hline $\mathcal{P}\left(\mathbb{R}^{N}\right):$ & the set of Borel probability measures on $\mathbb{R}^{N}$ \\
\hline$M(t):$ & the Monge functional (see $(1.1)$ \\
\hline $\mathcal{M}\left(\mu^{+}, \mu^{-}\right)$ & the set of transport maps between $\mu^{+}, \mu^{-}$ \\
\hline$K(\pi)$ & the Kantorovich functional (see $[1.2$ ) \\
\hline $\mathcal{P}\left(\mu^{+}, \mu^{-}\right)$ & the set of transport plans between $\mu^{+}, \mu^{-}$ \\
\hline$w_{p}\left(\mu^{+}, \mu^{-}\right)$ & theWasserstein distance of order $p$ between $\mu^{+}, \mu^{-}$ \\
\hline$W_{p}(\bar{X}):$ & theWasserstein space of order $p$ \\
\hline$\left(\Omega, \mathcal{B}(\Omega), \mu_{\Omega}\right)$ & the space of particles or reference space; $\mu_{\Omega}$ is the reference measure \\
\hline$\sigma_{\chi}(p)$ & the stopping time: $\sigma_{\chi}(p):=\inf \left\{t \in \mathbb{R}: \chi_{p}\right.$ const on $\left.[t,+\infty]\right\}$ \\
\hline$i_{\chi}(p):$ & the terminal point of the fiber $p: i_{\chi}(p):=\chi\left(p, \sigma_{\chi}(p)\right)=\chi(p, b)$ \\
\hline$\mu:$ & the irrigated measure: $\mu=i_{\chi \#} \mu_{\Omega} ;$ spt $\mu$ is compact, unless otherwise stated \\
\hline $\mathrm{AC}(I)$ : & the absolutely continuous functions on the interval $I$ \\
\hline$[p]_{t}^{i}, i=0,1,2$ & solidarity classes (see Definition 1.2 \\
\hline$m_{\chi}^{i}(p, t), i=0,1,2$ & a mass function (see 1.8 ) \\
\hline$s_{\alpha}^{i}(p, t), i=0,1,2$ & cost densities (see Definition 1.3 , \\
\hline$J_{\alpha}^{i}(p, t), i=0,1,2$ & cost functionals (see Definition 1.3 , \\
\hline$c(p):$ & the cost of the fiber $p($ see 2.1$)$ \\
\hline$D_{\chi}:$ & the domain of the pattern $\chi$ (see Definition 2.1 \\
\hline$Z_{\chi}:$ & see Definition 2.2 \\
\hline $\bar{\chi}:$ & the mass deviation of the pattern $\chi$ \\
\hline$\tilde{\chi}:$ & the mass by-pass of the pattern $\chi$ \\
\hline$d\left(x_{1}, x_{2}\right)$ : & the distance of $x_{1}, x_{2}$ on the fiber for a simple pattern (see 4.1 ) \\
\hline$\beta=1+h(\alpha-1):$ & the usual relation between $\beta, h, \alpha$ \\
\hline$l_{p}(x):$ & the distance of $x$ to the terminal point of the fiber $p$ (see $[7.2$ ) \\
\hline
\end{tabular}

\section{Notation}


$l(x)$ : the maximum distance of $x$ from a terminal point (see 7.1)

$d_{\alpha}(\mu, v):$

$\mathcal{H}^{\alpha}$ : the least cost of irrigation of $v$ from $\mu$ (see 3.3)

$\operatorname{dim}_{\mathcal{H}}(A)$ : the outer Hausdorff measure in dimension $\alpha$

$\operatorname{dim}_{\mathrm{c}}(\mu)$ : the Hausdorff dimension of the set $A$

$\operatorname{dim}_{M}(\mu)$ : $\operatorname{dim}_{\mathrm{res}}^{p}(\mu), \operatorname{dim}_{\mathrm{res}}^{\infty}(\mu)$ : the concentration dimension of the measure $\mu$ (see Definition A.1 the Minkowski dimension of the measure $\mu$ (see Definition A.3 the resolution dimension of the measure $\mu$ (see Definition A.7

\section{REFERENCES}

[A-V] Ambrosio, L., Caffarelli, L. A., Brenier, Y., Buttazzo, G., \& Villani, C. Optimal Transportation and Applications. Lecture Notes in Math. 1813, Springer, Berlin (2003). Zbl 1013.00028 MR 2006302

[BCM1] Bernot, M., Caselles, V., \& Morel, J.-M. Traffic plans. Publ. Mat. 49 (2005), 417-451. Zbl 1086.49029 MR 2177636

[BCM2] Bernot, M., CAselles, V., \& Morel, J.-M. Optimal Transportation Networks. Models and Theory. Lecture Notes in Math. 1955, Springer, Berlin (2009). Zbl 1163.90001 MR 2449900

[BF] Bernot, M., \& Figalli, A. Synchronized traffic plans and stability of optima. ESAIM Control Optim. Calc. Var. 14 (2008), 864-878. Zbl 1148.49039 MR 2451800

[DS1] Devillanova, G., \& Solimini, S. Elementary properties of optimal irrigation patterns. Calc. Var. Partial Differential Equations 28 (2007), 317-349. Zbl 1111.35093 MR 2290327

[DS2] Devillanova, G., \& Solimini, S. On the dimension of an irrigable measure. Rend. Sem. Mat. Univ. Padova 117 (2007), 1-49. Zbl 1165.28302 MR 2351784

[M] Mattila, P. Geometry of Sets and Measures in Euclidean Spaces. Fractals and Rectifiability. Cambridge Stud. Adv. Math. 44, Cambridge Univ. Press, Cambridge (1995). Zbl 0819.28004 MR 1333890

[MMS] Maddalena, F., Morel, J.-M., \& Solimini, S. A variational model of irrigation patterns. Interfaces Free Bound. 5 (2003), 391-415. Zbl 1057.35076 MR 2031464

[MS1] Maddalena, F., \& Solimini, S. Transport distances and irrigation models. J. Convex Anal. 16 (2009), 121-152. Zbl 1168.49042 MR 2531195

[MS2] Maddalena, F., \& Solimini, S. Synchronous and asynchronous descriptions of irrigation problems. Forthcoming.

[R] Royden, H. L. Real Analysis. 3rd ed., Macmillan, New York (1988). Zbl 0704.26006 MR 1013117

[S] Santambrogio, F. Optimal channel networks, landscape function and branched transport. Interfaces Free Bound. 9 (2007), 149-169. Zbl 1138.90339 MR 2317303

[V] Villani, C. Topics in Optimal Transportation. Grad. Stud. in Math. 58, Amer. Math. Soc., Providence, RI (2003). Zbl 1106.90001 MR 1964483

[X] XIA, Q. Optimal path related to transport problems. Comm. Contemp. Math. 5 (2003), 251-279. Zbl 1032.90003 MR 1966259 